\title{
धs \\ Theory of the superglass phase
}

\author{
Giulio Biroli, ${ }^{1}$ Claudio Chamon, ${ }^{2}$ and Francesco Zamponi ${ }^{3}$ \\ ${ }^{1}$ Institut de Physique Théorique, CEA, IPhT, F-91191 Gif-sur-Yvette, France and CNRS, URA 2306, F-91191 Gif-sur-Yvette, France \\ ${ }^{2}$ Physics Department, Boston University, Boston, Massachusetts 02215, USA \\ ${ }^{3}$ Laboratoire de Physique Théorique, École Normale Supérieure, CNRS-UMR 8549, 24 Rue Lhomond, 75231 Paris Cedex 05, France
}

(Received 22 July 2008; revised manuscript received 28 September 2008; published 8 December 2008)

\begin{abstract}
A superglass is a phase of matter which is characterized at the same time by superfluidity and a frozen amorphous structure. We introduce a model of interacting bosons in three dimensions that displays this phase unambiguously and that can be analyzed exactly or using controlled approximations. Employing a mapping between quantum Hamiltonians and classical Fokker-Planck operators, we show that the ground-state wave function of the quantum model is proportional to the Boltzmann measure of classical hard spheres. This connection allows us to obtain quantitative results on static and dynamic quantum correlation functions. In particular, by translating known results on the glassy dynamics of Brownian hard spheres we work out the properties of the superglass phase and of the quantum phase transition between the superfluid and the superglass phase.
\end{abstract}

DOI: 10.1103/PhysRevB.78.224306

PACS number(s): 61.43.Fs, 05.30.Jp, 67.80.K-

\section{INTRODUCTION}

Solids do not flow. This apparently tautological statement can be wrong in two ways. First, solidity is in general a time-scale-dependent phenomenon. Crystal or glasses, well known solids on most experimentally relevant time scales, do flow if one waits long enough (see Ref. 1, for example). Second, at very small temperatures, quantum solids may become superfluids as suggested theoretically in the early 1970 s. $^{2-4}$ A striking property of this phase of matter is the nonclassical rotational inertia: a supersolid placed at thermal equilibrium in a rotating container does not rotate, at least not completely, for small angular velocity $\omega$. In particular, its angular momentum is reduced from its classical value $I_{\mathrm{cl}} \omega$ by a fraction $f_{s}$ which is called superfluid fraction.

The first promising experimental evidence of supersolidity was found only four years ago by Chan and co-workers ${ }^{5,6}$ through measurements of a nonclassical rotational momentum of inertia (NCRI) in solid $\mathrm{He}^{4}$ at low temperature. Unfortunately, the physical mechanism underlying the observed effect is still unclear. In particular, despite intense experimental activity, it has not been established that the observed NCRI in solid $\mathrm{He}^{4}$ is accompanied by superflow (see, however, the very recent work reported in Ref. 7).

Nonetheless, some agreement has been reached by now on few basic facts: ${ }^{8}$ theoretical works have revealed that equilibrium $\mathrm{He}^{4}$ crystals at zero temperature are commensurate and not supersolids ${ }^{9-11}$ or at least have an extremely small superfluid fraction, too much small to account for experimental findings (see also Ref. 12). Experiments have shown that the supersolid critical temperature and the superfluid density depend sensitively on the detailed preparation history of the solid samples ${ }^{13}$ and on the presence of minute fractions of $\mathrm{He}^{3}$ impurities. ${ }^{5,6}$ The more disordered the $\mathrm{He}^{4}$ solids are the larger the supersolid signal is. It can reach surprisingly large values when the solid is formed by a rapid freezing from the normal phase. It can be reduced to an unobservable level by annealing. ${ }^{14}$ It is by now very plausible that rapid freezing produces highly disordered samples and that disorder is at the origin of the experimental findings and of "supersolid" signals. Different origins have been highlighted. A recent work in Ref. 15 suggested that the core of dislocations could be superfluid. A phenomenological theory of the role of dislocations can be found in Ref. 16 . Following up Ref. 17 a scenario to explain the experimental findings in terms of dislocation induced superfluidity has been put forward in Ref. 18. From the experimental point of view, the role of grain boundaries has been analyzed in Ref. 19. Another idea introduced in Refs. 20 and 21 is that rapid freezing produces amorphous glassy solids that become supersolid at low temperature. This "superglass" phase has been detected in path-integral Monte Carlo (PIMC) numerical simulations. ${ }^{20}$ These results are encouraging but addressing real-time dynamics issues such as the stability and the dynamics of this phase is out of reach of PIMC. Therefore, it is desirable to have a complementary analytical approach that can give direct insight into these problems: this is precisely the purpose of this work.

Our strategy will be to focus on a model that displays a superglass phase and that can be concretely analyzed in some instances exactly and in others using accurate and controlled approximations at zero temperature. The model consists of bosonic particles interacting via a short-range potential in three dimensions. Our approach is based on a mapping between quantum Hamiltonians and (classical) Fokker-Planck operators which allows us to obtain results on ground-state properties and time-dependent correlation functions from the analysis of the stochastic dynamics of a classical equilibrium system. This connection, already well known for a few decades, ${ }^{22,23}$ has been quite used and further studied in the context of the so-called Rokhsar-Kivelson points. ${ }^{24-26} \mathrm{We}$ will mainly focus on systems of interacting bosons whose classical counterpart (via the above mapping) is a system of hard spheres. These are known to undergo a crystal ${ }^{27}$ or a glass ${ }^{29-36}$ transition at high density. The glass transition takes place when crystallization is avoided (either by fast compression or using different types of particles). The usefulness of the classical-quantum mapping is that it allows us to obtain 
highly nontrivial, very accurate, or, at instances, exact results on the original quantum many-body problem by translating all the knowledge on the Brownian dynamics of hard spheres. In particular we will be able to work out in detail the properties of the superglass, supercrystal, and superfluid phases. The drawback of this mapping is that the resulting quantum solids are quite different from real $\mathrm{He}^{4}$ crystals mainly because of the much smaller zero-point motion and of some peculiar properties of the excitation spectrum to be discussed below.

The results of our work go beyond the physics of solid $\mathrm{He}^{4}$ because the superglass phase can emerge also in other physical contexts, in particular strongly interacting bosonic mixtures of cold atoms. Furthermore, another motivation for our study is to analyze a quantum glassy phase not induced by quenched disorder. There are, in fact, very few examples ${ }^{37-41}$ of that, especially not relying on mean-field or large- $N$ approximations.

\section{SUMMARY OF THE MAIN RESULTS}

In Sec. III the mapping between classical Fokker-Planck operators and quantum Hamiltonians is presented. In particular we will obtain the relation between the classical potential and its quantum counterpart. We will work it out for identical bosons as well as for mixtures of bosons (mixtures may be relevant for cold atomic systems). Furthermore, we shall introduce the model that we will focus on in this paper, which is the quantum counterpart of classical Brownian hard spheres. The quantum potential is still a hard sphere one but it has in addition a sticky part at contact (and also a threebody interaction at contact).

In Sec. IV we shall work out the zero-temperature phase diagram of the quantum model as a function of the density $\rho$. Actually we will use instead of the density the packing fraction $\phi=\pi \rho \sigma^{3} / 6$ (where $\sigma$ is the sphere diameter) which is a more natural variable for hard spheres. We find three possible phases: superfluid, supercrystal, and superglass. At low packing fraction the system is superfluid but at $\phi \simeq 0.49$ it has a first-order transition toward a supercrystal phase. The transition toward the superglass phase takes place at higher packing fractions, $\phi \simeq 0.62$. It can be achieved only if the transition toward the crystal is avoided either by considering mixtures or compressing fast the liquid phase. We will obtain quantitative results for the condensate fraction. In particular for the superglass phase we will introduce and compute an observable, akin to the Edwards-Anderson parameter for spin glasses, which quantifies the inhomogeneity of the condensate wave function in space. The values of the condensate fraction turn out to be very small. This is likely due to the particular model we have chosen which admits as groundstate wave function the Gibbs measure of hard spheres. However, exact results guarantee that, although small, the condensate fraction is nonzero, i.e., it is not an artifact. In Sec. V we focus on the real-time quantum dynamics close to the transition from the superfluid to the superglass. In particular we obtain the behavior of the density correlations as a function of time as well as the time-dependent condensate fluctuations. Both correlation functions show a similar behavior.
Approaching the superglass from the superfluid, one finds that after a rapid oscillating decay, there is a plateau indicating frozen amorphous density and condensate fluctuations. On much larger time scales these frozen fluctuations relax. The transition to the superglass phase takes place when the time scale for this second relaxation goes to infinity (or, practically, becomes larger than any relevant experimental time scale). This behavior is actually very reminiscent of the one of the classical supercooled liquids approaching their structural glass transition. In Sec. VI we will generalize our model in order to make it more realistic. In fact, within the classical-quantum-mapping approach, the energy of the ground state is always zero. In order to discuss the behavior of the pressure, the order of the transitions, and the superfluid properties we shall add a small perturbation to the quantum potential originally introduced in Sec. III. This will allow us to show that the transition from the superfluid to the superglass is unusual to the extent that it is thermodynamically first order but has also some characteristics of secondorder transitions. Finally, conclusions, perspectives, and relations to experiments and numerical simulations will be presented in Sec. VII.

\section{FROM CLASSICAL LANGEVIN PARTICLES TO ZERO TEMPERATURE INTERACTING BOSONS}

The superglass is an amorphous quantum many-body state of interacting bosons. To find such a state starting from a generic quantum Hamiltonian of interacting particles is a daunting task. In this paper, we take an approach that allows us to argue for such a phase in a well controlled way by constructing a local quantum many-body Hamiltonian whose ground state is known exactly and can be argued to be a glassy state at large enough densities. This formulation is motivated by a generic result stating that for systems without a sign problem there is a simple connection between quantum Hamiltonians and the stochastic dynamics of a classical system. This can be used to construct interesting Hamiltonians with real and non-negative ground-state wave functions related to classical equilibrium Boltzmann-Gibbs measures. The connection is far reaching since it allows one to obtain controlled and highly nontrivial results on the phase diagram and dynamical properties of a quantum many-body problem. ${ }^{26}$ Moreover, it has been used to construct an efficient algorithm for quantum Monte Carlo at zero temperature. ${ }^{42}$ Here we shall explore this classical-quantum connection for the particular case of bosonic point particles, following the standard route for mapping classical Langevin dynamics for a many-particle system and its associated Fokker-Planck operator to a Schrödinger operator. ${ }^{22,23}$

\section{A. Langevin dynamics: The Fokker-Planck and Schrödinger operators}

Consider $N$ particles whose evolution is determined by the following Langevin equations:

$$
\gamma_{i} \frac{d \mathbf{x}_{i}}{d t}=-\frac{\partial}{\partial \mathbf{x}_{i}} U_{N}\left(\mathbf{x}_{1}, \ldots, \mathbf{x}_{N}\right)+\boldsymbol{\eta}_{i}(t), \quad i=1, \ldots, N,
$$

where $\gamma_{i}$ are friction coefficients and $\eta_{i}^{\alpha}(t)$ is a Gaussian white thermal noise with variance $\left\langle\eta_{i}^{\alpha}(t) \eta_{j}^{\beta}\left(t^{\prime}\right)\right\rangle$ 
$=2 T \gamma_{i} \delta_{i j} \delta_{\alpha \beta} \delta\left(t-t^{\prime}\right)$. Furthermore, $T$ is the temperature (with $\left.k_{B}=1\right)$ and $\alpha$ and $\beta$ run from one to the spatial dimension $d$ (henceforth the boldface notation indicates vectors). The potential will eventually be assumed to be the sum of (symmetric) pair potentials,

$$
U_{N}(\{\mathbf{x}\}) \equiv U_{N}\left(\mathbf{x}_{1}, \ldots, \mathbf{x}_{N}\right)=\frac{1}{2} \sum_{i \neq j} V_{i j}\left(\mathbf{x}_{i}-\mathbf{x}_{j}\right),
$$

with $V_{i j}=V_{j i}$ and $\frac{\partial}{\partial \mathbf{x}_{i}} U_{N} \equiv \nabla_{i} U_{N}=\sum_{j(\neq i)} \nabla V_{i j}\left(\mathbf{x}_{i}-\mathbf{x}_{j}\right)$.

It is well known ${ }^{22,23}$ that the evolution equation for the probability distribution $P(\{\mathbf{x}\})$ can be written as a Schrödinger equation in imaginary time,

$$
\partial_{t} P=-H_{\mathrm{FP}} P,
$$

where the Fokker-Planck operator reads

$$
H_{\mathrm{FP}}=-\sum_{i} \frac{1}{\gamma_{i}} \frac{\partial}{\partial \mathbf{x}_{i}}\left[\nabla_{i} U_{N}+T \frac{\partial}{\partial \mathbf{x}_{i}}\right] .
$$

The Fokker-Planck operator is non-Hermitian and can be proven to have all eigenvalues larger than or equal to zero. $^{22,23}$ The zero eigenvalue corresponds-as it can be readily checked-to the stationary distribution which is the equilibrium Gibbs probability measure,

$$
P_{G}(\{\mathbf{x}\})=\frac{1}{Z_{N}} e^{-(1 / T) U_{N}(\{\mathbf{x}\})}=\frac{1}{Z_{N}} \exp \left[-\frac{1}{2 T} \sum_{i \neq j} V_{i j}\left(\mathbf{x}_{i}-\mathbf{x}_{j}\right)\right] .
$$

Setting for simplicity $\hbar=1$, the Fokker-Planck operator can be mapped into a Hermitian quantum Hamiltonian by a similarity transformation,

$$
H=e^{(1 / 2 T) U_{N}} H_{\mathrm{FP}} e^{-(1 / 2 T) U_{N}},
$$

which leads to

$$
\begin{aligned}
H & =\sum_{i} \frac{1}{\gamma_{i}}\left[-T \frac{\partial^{2}}{\partial \mathbf{x}_{i}^{2}}-\frac{1}{2} \nabla_{i}^{2} U_{N}+\frac{1}{4 T}\left(\nabla_{i} U_{N}\right)^{2}\right] \\
& =\sum_{i} \frac{\mathbf{p}_{i}^{2}}{2 m_{i}}+\mathcal{V}_{N}(\{\mathbf{x}\}) .
\end{aligned}
$$

This expression corresponds to a Hamiltonian for particles with mass $m_{i}=\gamma_{i} /(2 T)$ and an effective potential which is the sum of two-body and three-body interactions,

$$
\begin{aligned}
\mathcal{V}_{N}(\{\mathbf{x}\})= & \sum_{i} \frac{1}{\gamma_{i}}\left[-\frac{1}{2} \nabla_{i}^{2} U_{N}+\frac{1}{4 T}\left(\nabla_{i} U_{N}\right)^{2}\right] \\
= & -\frac{1}{2} \sum_{j \neq i} \frac{1}{\gamma_{i}} \nabla^{2} V_{i j}\left(\mathbf{x}_{i}-\mathbf{x}_{j}\right) \\
& +\frac{1}{4 T} \sum_{i ; j(\neq i) ; j^{\prime}(\neq i)} \frac{1}{\gamma_{i}} \nabla V_{i j}\left(\mathbf{x}_{i}-\mathbf{x}_{j}\right) \cdot \nabla V_{i j^{\prime}}\left(\mathbf{x}_{i}-\mathbf{x}_{j^{\prime}}\right) .
\end{aligned}
$$

The eigenfunctions of the quantum Hamiltonian and of the Fokker-Planck operator are in a one-to-one correspondence.
Indeed, by applying the similarity transformation introduced above one finds

$$
\Psi_{E}(\{\mathbf{x}\}) \propto e^{U_{N} / 2 T} P_{E}(\{\mathbf{x}\}),
$$

where $P_{E}$ indicates the right eigenfunction of the FokkerPlanck operator with eigenvalue $E$ and $\Psi_{E}$ its counterpart associated to the quantum Hamiltonian. This also implies that all the eigenvalues $E$ corresponding to the FokkerPlanck operator are identical to the ones of the quantum Hamiltonian.

In particular, this relation, together with Eq. (5), allows one to obtain straightforwardly the ground-state wave function of the quantum problem, which is of the Jastrow form, ${ }^{43-45}$

$$
\Psi_{G}(\{\mathbf{x}\})=\sqrt{P_{G}(\{\mathbf{x}\})}=\frac{1}{\sqrt{Z_{N}}} \exp \left[-\frac{1}{4 T} \sum_{i \neq j} V_{i j}\left(\mathbf{x}_{i}-\mathbf{x}_{j}\right)\right] .
$$

The logic of the approach we pursue hereafter is the following: we take as starting point Hamiltonians with many-body potentials of form (8), for which the Jastrow form ${ }^{43}$ [Eq. (10)] is exact. In general, wave functions of this form lead to more than two-body interactions $\mathcal{V}_{N}(\{\mathbf{x}\})$ (note that also $\mathrm{He}^{4}$ has weak higher order interactions). The important point is that if the two-body potentials $V_{i j}\left(\mathbf{x}_{i}-\mathbf{x}_{j}\right)$ are short-ranged (i.e., local) potentials, then $\mathcal{V}_{N}(\{\mathbf{x}\})$ is also local and thus the many-body Hamiltonians on which we focus are local (nonlocal Hamiltonian may lead to pathological behaviors).

Because we know exactly the ground-state wave function, and it is related to a Boltzmann-Gibbs measure for a classical system, quantum static correlation functions can be computed in terms of classical static correlation functions. ${ }^{45}$ Furthermore, as we shall show and noticed by Henley, ${ }^{25}$ the mapping generalizes also to dynamical correlation functions. Hence, we will obtain quantum dynamical correlation functions at zero temperature by analytic continuation of classical (stochastic) dynamical correlation functions.

To simplify the notations, in the following we will fix $T$ $=1$ and $\langle\gamma\rangle=N^{-1} \sum_{i} \gamma_{i}=1$. Together with $\hbar=1$, this fixes the units in both classical and quantum problems. As a consequence the masses in the quantum problem read $m_{i}=\frac{1}{2} \frac{\gamma_{i}}{\langle\gamma\rangle}$.

\section{B. Identical bosons}

Let us first consider the simplest case of $N$ identical bosons characterized by the Hamiltonian $H$ in Eq. (7) with $\gamma_{i} \equiv \gamma=1$ and $V_{i j} \equiv V$. It is important to remark that since the particles are bosons one has to consider only many-body states that are completely symmetric under permutation of particles and study only observables that are invariant under permutation of particles (e.g., the density-density correlator). This is clearly not a difficult constraint to handle since Jastrow form (10) (Ref. 43) with $V_{i j}=V$ is completely symmetric. Furthermore, even in the study of dynamical correlations (Sec. V) this will not be a problem because if one starts from a probability law completely symmetric under permutation of particles, the symmetrization carries over to all later times. This follows trivially from the Fokker-Planck evolution [Eq. 
(3)] since if the state $P$ and the operator $H_{\mathrm{FP}}$ are both symmetric under exchange of particles, so is the time derivative $\partial_{t} P$ and thus the many-body state thereafter.

For a given classical isotropic two-body potential $V(\mathbf{x})$ $=V(|\mathbf{x}|)$, the resulting quantum potential energy will have two-body and three-body interactions,

$$
\begin{aligned}
\mathcal{V}_{N}(\{\mathbf{x}\})= & \sum_{i>j} v^{\text {pair }}\left(\mathbf{x}_{i}-\mathbf{x}_{j}\right) \\
& +\sum_{i \neq j \neq j^{\prime} \neq i} v^{\text {three body }}\left(\mathbf{x}_{i}-\mathbf{x}_{j}, \mathbf{x}_{i}-\mathbf{x}_{j^{\prime}}\right), \\
v^{\text {pair }}(\mathbf{x})= & -\nabla^{2} V(\mathbf{x})+\frac{1}{2}[\nabla V(\mathbf{x})]^{2} \\
= & -\frac{d-1}{r} V^{\prime}(r)-V^{\prime \prime}(r)+\frac{1}{2}\left[V^{\prime}(r)\right]^{2}, \\
v^{\text {three body }}\left(\mathbf{x}, \mathbf{x}^{\prime}\right)= & \frac{1}{4} \nabla V(\mathbf{x}) \cdot \nabla V\left(\mathbf{x}^{\prime}\right)=\frac{1}{4} \frac{\mathbf{x}}{r} \cdot \frac{\mathbf{x}^{\prime}}{r^{\prime}} V^{\prime}(r) V^{\prime}\left(r^{\prime}\right),
\end{aligned}
$$

where $d$ is the spatial dimension and $r \equiv|\mathbf{x}|$.

\section{Binary mixtures of bosons}

It is also worthwhile considering binary mixtures of bosons. In fact, the superglass phase we shall discuss in the following will emerge only when crystallization is avoided. We expect, as it is the case for classical systems, that this can be obtained either by fast compression of the liquid state or by considering mixtures of bosons. Mixtures typically induce a frustration effect on the crystalline phase whose energy, as a consequence, increases. Instead the glass phase, thanks to its disordered structure, is less affected and, hence, starts to compete in terms of energy (or dynamical basin of attraction) with the crystalline phase. This conclusion, which we expect to be correct on physical grounds, is also suggested by approaches based on variational wave functions of the Jastrow form $[\mathrm{Eq} .(10)] .{ }^{43}$ These types of wave functions map the quantum problem into a classical one and are very effective in describing a number of the observed phenomena in the quantum Bose fluid. Lennard-Jones-type potentials (as classical potentials in the exponent of the Jastrow wave function $^{43}$ ) have been considered by McMillan ${ }^{44}$ and Francis et $a l .{ }^{45}$ in the study of $\mathrm{He}^{4}$ superfluids. For a single species of particles, such interaction potentials lead to classical liquid states and, at very high densities, might lead to a crystalline state. ${ }^{46}$ Within Jastrow-type approaches, ${ }^{43}$ thanks to this analogy with classical systems, we expect the physical effect induced by mixtures to be similar to what happens in classical liquids.

As a consequence, we conjecture that for a binary mixture, a quantum glassy state may emerge at high enough densities. Experimentally, the binary mixture can be obtained using two species of bosonic atoms, $A$ and $B$. It may be the case that we do not need to develop a condensate fraction for both species $A$ and $B$; it would suffice to have a superglass

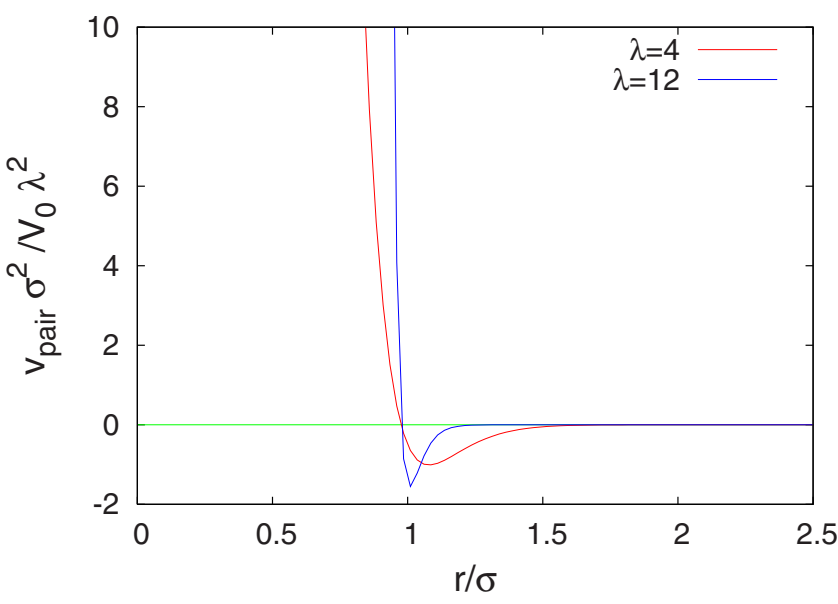

FIG. 1. (Color online) Form of the pair potential $v^{\text {pair }}(r)$ in the mapped quantum problem that derives from the classical potential $V(r)=V_{0} \exp \left(-\lambda\left[(r / \sigma)^{2}-1\right]\right)$. (Notice that we have set $V_{0}=1$ and $\sigma=1$ in the text.)

of, say, species $A$, while species $B$ always remains normal, with its role solely that of enabling the formation of the glassy state. This type of binary mixture can be obtained in cold atom experiments.

The generalization of the results of Sec. III B to a binary mixture is presented in Appendix. In the following we will focus on the identical boson system but one must keep in mind that to stabilize the glassy phase one may have to consider mixtures or fast compression of the liquid state. Thus, our strategy will be to study directly the glass phase of identical particles and translate the results to the mixture case where this phase actually exists.

\section{Quantum model with hard sphere wave function}

Let us investigate what quantum bosonic system in particular corresponds to a classical hard sphere problem upon the mapping above. The motivation for this study is that the classical hard sphere packing is a well-studied problem in the context of glasses for large enough packing fractions, ${ }^{29-36}$ and we will be able to use these results for substantiating the notion of the superglass state. We shall focus on a classical potential $V(r)=V_{0} \exp \left(-\lambda\left[(r / \sigma)^{2}-1\right]\right)$, where $\sigma$ is the characteristic sphere size, perform the mapping, and take the $\lambda$ $\rightarrow \infty$ limit that enforces the hard sphere constraint. Note that although in principle the form of the potential is not very important as long as it is infinite for $r<\sigma$ and zero for $r$ $>\sigma$ (in the $\lambda \rightarrow \infty$ limit), the previous form makes the following discussion particularly easy. We also set $V_{0}=1$ and $\sigma=1$ for simplicity; indeed, even if the units have already been fixed, $V_{0}$ is irrelevant in the $\lambda \rightarrow \infty$ limit, while a change in $\sigma$ is equivalent to a change in the density.

Let us first discuss the pair term, which depends only on the interparticle distance $r$,

$$
v^{\text {pair }}(r)=\left[2 \lambda d-4 \lambda^{2} r^{2}\right] V(r)+2 \lambda^{2} r^{2}[V(r)]^{2},
$$

where we have used explicitly the (convenient) exponential form of the potential. In Fig. 1 we show the form of this two-body potential. 
The form of the pair potential in the large $\lambda$ limit is simple.

(i) For $r>1$, the pair potential is zero because the exponential terms go very fast to zero.

(ii) For $r<1$, the pair potential goes to infinity. Although the second term is negative, the third one is much larger than the first two and dominates.

(iii) Very close to $r=1$, within a window that shrinks to zero in the large $\lambda$ limit, the potential becomes negative and goes to minus infinity. Indeed, for $r=1$, its value is $2 d \lambda$ $-2 \lambda^{2}$. The potential remains attractive (negative) on an interval of size $1 / \lambda$. This attractive part of the potential is responsible for the fact that ground-state wave function does not vanish (or is discontinuous) at the contact between hard spheres. Particles can stay close to contact paying a lot of kinetic energy, $\mathcal{O}\left(\lambda^{2}\right)$, and gaining a lot of potential energy, also $\mathcal{O}\left(\lambda^{2}\right)$.

Let us now consider the three-body term. It can be written as

$$
v^{\text {three body }}\left(\mathbf{x}, \mathbf{x}^{\prime}\right)=\lambda^{2} \mathbf{x} \cdot \mathbf{x}^{\prime} V(r) V\left(r^{\prime}\right),
$$

where for a given triplet of particles $i, j, j^{\prime}$ one has $\mathbf{x}=\mathbf{x}_{i}$ $-\mathbf{x}_{j}$ and $\mathbf{x}^{\prime}=\mathbf{x}_{i}-\mathbf{x}_{j^{\prime}}$ [see Eq. (11)]. The form of the threebody potential in the large $\lambda$ limit is simple: it is nonzero only if both particles $j$ and $j^{\prime}$ have a nonzero overlap with particle $i$. When there is a finite overlap (in the large $\lambda$ limit) the contribution coming from the three-body term, which can be actually positive or negative, is always smaller than the one coming from the sum of the pair contributions $i j$ and $i j^{\prime}$. Thus, it can be neglected. The outcome coming from the pair interactions is that all particle configurations with finite particle overlaps are simply excluded by the Hilbert space. When both $r$ and $r^{\prime}$ are very close to 1 , of the order $1 / \lambda$, the three-body term will give rise to a non-negligible contribution similar to the one studied for the pair contribution. The main difference is that it can be attractive or repulsive depending on the relative orientation of $\mathbf{x}$ and $\mathbf{x}^{\prime}$. As a conclusion the interaction between particles is the hard sphere one plus a contact term that is sticky for the pair contribution and that can be repulsive or attractive for the triplet term depending on the geometry of the triplet.

\section{SUPERFLUID, SUPERCRYSTAL, AND SUPERGLASS PHASES}

Here we make use of the relationship between the classical and quantum models and study the zero-temperature phases of the quantum system introduced in Sec. III D. We will work out the properties of the quantum phases by using the classical Gibbs measure defined by the square of the Jastrow wave function. ${ }^{26,43-46}$

We shall focus on a system of $N$ identical bosons with the particular interaction, discussed in Sec. III D, which corresponds to a Jastrow state ${ }^{43}$ with a hard sphere potential. Having fixed $\sigma=1$, the control parameter in this case is the particle density $\rho=N / V$ of the particles or the packing fraction $\phi=\pi \rho / 6$. Let us recall first the phase diagram of classical hard spheres ${ }^{27,34,47}$ (see top of Fig. 2). At low density, the system is liquid, and upon increasing the density it undergoes

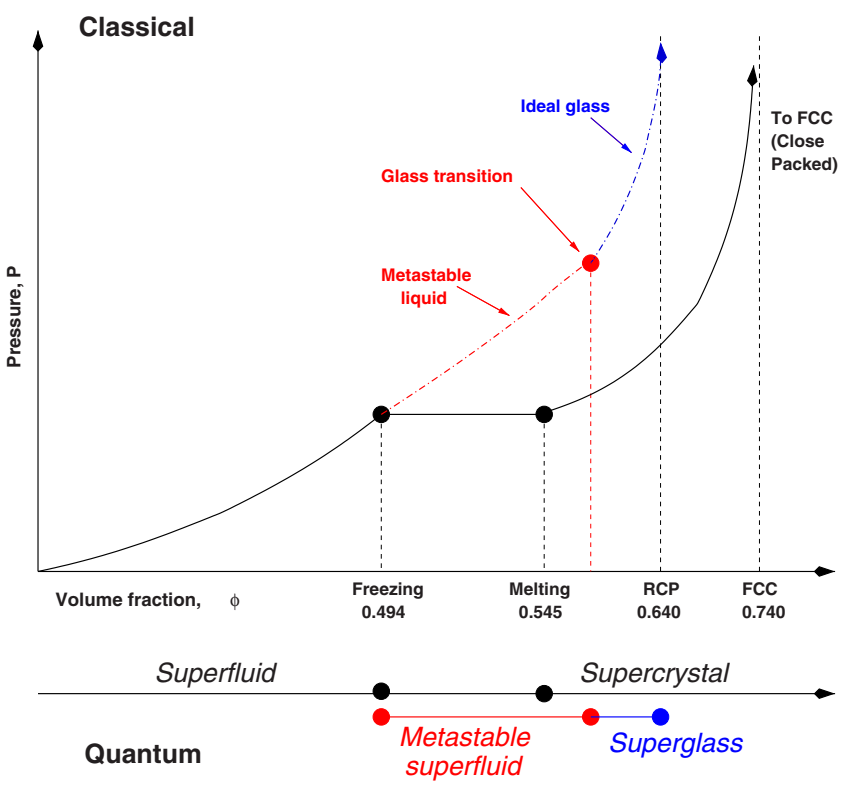

FIG. 2. (Color online) Classical phase diagram for hard sphere systems (top) and its quantum counterpart (bottom).

a first-order phase transition to a crystalline state, which is arranged in a face-centered-cubic (fcc) lattice. Moreover, a metastable glassy phase can be obtained in the classical problem if the density is increased fast enough or in presence of small bidispersity (binary mixtures). This glass phase can be compressed until the random close packing (RCP) fraction $\phi \sim 0.64$. These three classical phases have their corresponding counterparts in the mapped quantum system. As we shall show, these are superfluid, supercrystal, and superglass phases. The classical phase diagram and its quantum counterpart are shown in Fig. 2.

Below we will discuss the property of these three quantum phases in some detail with particular emphasis on their one-particle density matrix and Bose condensate fraction. We will treat the glass phase as a true equilibrium phase because, as we have already discussed, it will emerge when crystallization is avoided either by fast compression of the liquid state or considering suitable binary mixtures.

\section{A. Off-diagonal long-range order and classical correlation functions}

Let us first recall general results on off-diagonal longrange order (ODLRO) ${ }^{48,49}$ In particular, we shall show that for Jastrow-type wave functions ${ }^{43}$ there is a simple relation ${ }^{44,45,48}$ between the structure factor of a classical system and the one-particle density matrix. This relation has a particularly simple form in the case of hard spheres. The condensate fraction is related to the amount of off-diagonal long--range order in the quantum problem and can be therefore related to an observable for the classical corresponding liquid. In Appendix, Sec. 2 we generalize the following discussion of ODLRO to the case of binary mixtures.

The one-particle off-diagonal density matrix reads in term of the ground-state wave function $\Psi_{G}$ ( $V$ is the volume), 


$$
\begin{aligned}
\mathcal{R}\left(\mathbf{x}, \mathbf{x}^{\prime}\right)= & V \int d \mathbf{x}_{2}, \ldots, d \mathbf{x}_{N} \Psi_{G}\left(\mathbf{x}, \mathbf{x}_{2}, \ldots, \mathbf{x}_{N}\right) \\
& \times \Psi_{G}\left(\mathbf{x}^{\prime}, \mathbf{x}_{2}, \ldots, \mathbf{x}_{N}\right) \\
= & V \sum_{i} n_{i} \psi_{i}(\mathbf{x}) \psi_{i}^{*}\left(\mathbf{x}^{\prime}\right),
\end{aligned}
$$

where the $n_{i}$ and $\psi_{i}(\mathbf{x})$ are, respectively, eigenvalues and (orthonormal) eigenvectors of the one-particle density matrix $\mathcal{R}\left(\mathbf{x}, \mathbf{x}^{\prime}\right), \int d \mathbf{x}^{\prime} \mathcal{R}\left(\mathbf{x}, \mathbf{x}^{\prime}\right) \psi_{i}\left(\mathbf{x}^{\prime}\right)=n_{i} \psi_{i}(\mathbf{x})$ (see, e.g., Ref. 49). One can interpret $n_{i}$ as the fraction of particles in state $i$, and $\Sigma_{i} n_{i}=1$. The largest eigenvalue $n_{0}$ gives directly the condensate fraction.

If $\Psi_{G}$ is given by a Jastrow wave function ${ }^{43}$ with a hard sphere potential, this expression is particularly simple in terms of a classical equilibrium correlation function,

$$
\begin{aligned}
\mathcal{R}\left(\mathbf{x}, \mathbf{x}^{\prime}\right) & =\frac{V}{N(N+1)} \frac{Z_{N+1}}{Z_{N}} e^{V\left(\mathbf{x}-\mathbf{x}^{\prime}\right) / T} \sum_{i \neq j}\left\langle\delta\left(\mathbf{x}-\mathbf{x}_{i}\right) \delta\left(\mathbf{x}^{\prime}-\mathbf{x}_{j}\right)\right\rangle \\
& =\frac{Z_{N+1}}{Z_{N}(N+1)} \rho^{-1} \rho(\mathbf{x}) \rho\left(\mathbf{x}^{\prime}\right) y\left(\mathbf{x}, \mathbf{x}^{\prime}\right),
\end{aligned}
$$

where $\rho(\mathbf{x})=\left\langle\sum_{i} \delta\left(\mathbf{x}-\mathbf{x}_{i}\right)\right\rangle, \quad y\left(\mathbf{x}, \mathbf{x}^{\prime}\right)=e^{V\left(\mathbf{x}-\mathbf{x}^{\prime}\right) / T} g\left(\mathbf{x}, \mathbf{x}^{\prime}\right), \quad$ and $g\left(\mathbf{x}, \mathbf{x}^{\prime}\right)$ is the usual pair correlation function. ${ }^{50}$ Notice that this expression follows by considering a system of $N+1$ particles, with two of them fixed at $\mathbf{x}$ and $\mathbf{x}^{\prime}$, and that the difference between $y\left(\mathbf{x}, \mathbf{x}^{\prime}\right)$ and $g\left(\mathbf{x}, \mathbf{x}^{\prime}\right)$ arises because $\mathcal{R}\left(\mathbf{x}, \mathbf{x}^{\prime}\right)$ does not vanish when $\mathbf{x}$ and $\mathbf{x}^{\prime}$ are less than a particle diameter away. For hard spheres, there is a simplification and $g\left(\mathbf{x}, \mathbf{x}^{\prime}\right)=y\left(\mathbf{x}, \mathbf{x}^{\prime}\right)$ for $\left|\mathbf{x}-\mathbf{x}^{\prime}\right|>1$; however, for $\left|\mathbf{x}-\mathbf{x}^{\prime}\right|<1$ the pair correlation function vanishes while $y\left(\mathbf{x}, \mathbf{x}^{\prime}\right)$ is finite. ${ }^{50}$

The prefactor in Eq. (15) is related to the fugacity of the system and it is easily evaluated in the large $N$ limit,

$$
\begin{aligned}
Z_{N} & \sim N ! e^{N S(N / V)} \Rightarrow \frac{Z_{N+1}}{Z_{N}(N+1)} \\
& =e^{(d / d \rho)[\rho S(\rho)]}=\rho^{-1} e^{(d / d \rho)\left[\rho S_{\mathrm{ex}}(\rho)\right]} \equiv \rho^{-1} f(\rho)=\frac{1}{z(\rho)},
\end{aligned}
$$

where $S(\rho)=1-\log \rho+S_{\mathrm{ex}}(\rho)$ is the entropy of classical hard spheres as a function of the average density $\rho=N / V, S_{\text {ex }}(\rho)$ is the excess entropy with respect to the ideal gas, and $z(\rho)$ is the fugacity. Using this relation we find the final, quite simple, expression,

$$
\mathcal{R}\left(\mathbf{x}, \mathbf{x}^{\prime}\right)=f(\rho) \frac{\rho(\mathbf{x}) \rho\left(\mathbf{x}^{\prime}\right)}{\rho^{2}} y\left(\mathbf{x}, \mathbf{x}^{\prime}\right) .
$$

For a homogeneous phase, $\rho(\mathbf{x})=\rho$ and $\mathcal{R}\left(\mathbf{x}, \mathbf{x}^{\prime}\right)$ becomes a function of $\left|\mathbf{x}-\mathbf{x}^{\prime}\right|$ only,

$$
\mathcal{R}\left(\left|\mathbf{x}-\mathbf{x}^{\prime}\right|\right)=f(\rho) y\left(\left|\mathbf{x}-\mathbf{x}^{\prime}\right|\right),
$$

and the condensate fraction is just $\lim _{\left|\mathbf{x}-\mathbf{x}^{\prime}\right| \rightarrow \infty} \mathcal{R}\left(\left|\mathbf{x}-\mathbf{x}^{\prime}\right|\right)$ $=f(\rho)$ since $y\left(\mathbf{x}, \mathbf{x}^{\prime}\right) \rightarrow 1$ (like the pair correlation function) as $\left|\mathbf{x}-\mathbf{x}^{\prime}\right| \rightarrow \infty$. This expression shows that as long as the fugacity is finite, i.e., the pressure is finite, we shall find a nonzero condensate fraction. ${ }^{48}$ As a consequence, we expect a superfluid phase at low densities, then a first-order transition into a supersolid crystalline phase as the density is increased. In the case of nonidentical hard spheres, e.g., bidisperse, or for extremely rapid quenches, the system should end up in a superglass phase at high density, and the condensate fraction will vanish only at close packing. In the following, we will apply the results above and study in detail the superfluid, supercrystal, and superglass phases.

\section{B. Superfluid phase}

At low packing fraction the classical system is in the liquid phase. Analogously, the quantum system is in a quantum liquid phase. As discussed above, we expect it to be superfluid. In the following we shall compute its corresponding condensate fraction. In order to obtain a heuristic semiquantitative expression for the condensate fraction, we used the Carnahan-Starling approximation ${ }^{50}$ for the classical hard sphere system. This is a kind of virial resummation known to work very well for describing the properties of the hard sphere system in the whole fluid range. Within this approximation one finds ${ }^{50}$

$$
\rho S_{\mathrm{ex}}(\rho)=\frac{1}{V} \log \left(Z_{N} / V^{N}\right)=-\frac{6 \phi}{\pi} \frac{\phi(4-3 \phi)}{(1-\phi)^{2}} .
$$

Then Eq. (16) leads to the following expression for the condensate fraction $n_{0}$ :

$$
n_{0}=f(\rho)=\exp \left[\frac{\phi\left(-8+9 \phi-3 \phi^{2}\right)}{(1-\phi)^{3}}\right] .
$$

This is plotted in Fig. 3. Note that the freezing crystallization transition takes place at a packing fraction $\phi \simeq 0.494$ for hard spheres (see Fig. 2).

\section{Crystal phase}

For $\phi>0.545$, the classical hard sphere system is in the crystal phase (for $0.494<\phi<0.545$ there is phase coexistence between the liquid and the crystal). Correspondingly, the quantum system displays a quantum crystal phase. In the following we shall compute its condensate fraction. The crystal phase is inhomogeneous but still $y\left(\mathbf{x}, \mathbf{x}^{\prime}\right) \rightarrow 1$ at large $\left|\mathbf{x}-\mathbf{x}^{\prime}\right|$; therefore we have ${ }^{48}$

$$
\lim _{\left|\mathbf{x}-\mathbf{x}^{\prime}\right| \rightarrow \infty} \mathcal{R}\left(\mathbf{x}, \mathbf{x}^{\prime}\right)=f(\rho) \frac{\rho(\mathbf{x}) \rho\left(\mathbf{x}^{\prime}\right)}{\rho^{2}}=n_{0} V \psi_{0}(\mathbf{x}) \psi_{0}^{*}\left(\mathbf{x}^{\prime}\right),
$$

and from this factorization one obtains that $\sqrt{n_{0} V} \psi_{0}(\mathbf{x})$ $=\sqrt{f(\rho)} \rho(\mathbf{x}) / \rho$ [notice that the eigenvector $\psi_{0}(\mathbf{x})$ is real valued, as $\mathcal{R}\left(\mathbf{x}, \mathbf{x}^{\prime}\right)$ is real and symmetric]. It thus follows from the normalization of $\psi_{0}(\mathbf{x})$ that

$$
n_{0}=f(\rho) \frac{1}{\rho^{2}} \frac{1}{V} \int d \mathbf{x} \rho(\mathbf{x})^{2} .
$$

A rough estimate of $n_{0}$ is given by $f(\rho)$ since $f(\rho) \leq n_{0}$ $\leq\left(\max _{V} \frac{\rho(\mathbf{x})}{\rho}\right)^{2} f(\rho)$. The first inequality follows from $\rho^{2}$ $=\left[\frac{1}{V} \int d \mathbf{x} \rho(\mathbf{x})\right]^{2} \leq \frac{1}{V} \int d \mathbf{x} \rho(\mathbf{x})^{2}$. However, because of the inhomogeneity of the density profile, the contribution to $n_{0}$ com- 


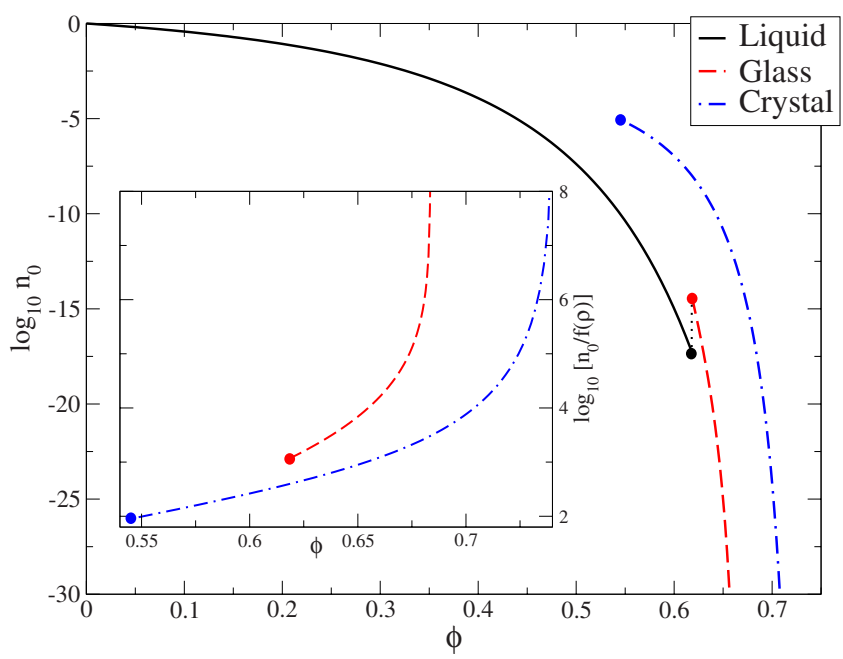

FIG. 3. (Color online) Condensate fraction as a function of the packing fraction for the hard sphere Jastrow wave function (Ref. 43). Full (black) curve: superfluid phase [Eq. (20)]. Dotted-dashed (blue/dark gray) curve: supercrystal phase [Eq. (24)]. Dashed (red/ gray) curve: superglass phase [Eq. (39)]; note that the theory slightly overestimates the random close packing density with respect to the commonly accepted value of $\phi=0.64$. The blue/dark gray dot marks the location of the melting transition, where liquidcrystal phase coexistence begins, while the red/gray dot marks the glass transition density. Inset: the enhancement factor $n_{0} / f(\rho)$ due to the spatial inhomogeneity of the crystal and glass phases. Note that this factor is quite large, of the order of $10^{2}-10^{3}$ at the melting and glass transition densities. Still, the condensate fractions we find are extremely small, probably due to the classical-like nature of solids with the Jastrow hard sphere wave function (Ref. 43).

ing from the integral of the density squared may be quite large. In order to estimate it we assume that

$$
\rho(\mathbf{x})=\sum_{i} \frac{e^{-\left|\mathbf{x}-\mathbf{R}_{i}\right|^{2} / 2 A}}{(2 \pi A)^{3 / 2}},
$$

where $\mathbf{R}_{i}$ are the fcc lattice sites and the Gaussians are roughly nonoverlapping. It has been shown by numerical simulations that this approximation is rather $\operatorname{good}^{51}$ and values of $A$ have been computed by density functional theory. ${ }^{52}$ Using these results one finds

$$
n_{0}=f(\rho) \frac{1}{\rho^{2}} \frac{1}{V} \int d \mathbf{x} \rho(\mathbf{x})^{2}=\frac{f(\rho)}{\rho} \frac{1}{(4 \pi A)^{3 / 2}},
$$

with $f(\rho)$ given in Eq. (16).

To compute $f(\rho)$ we used the phenomenological equation of state for the fcc crystal phase of classical hard spheres proposed by Speedy, ${ }^{53}$

$$
\frac{P}{\rho}=\frac{3}{1-z}-a \frac{z-b}{z-c}, \quad z=\phi / \phi_{\mathrm{fcc}},
$$

where $\phi_{\mathrm{fcc}}=\pi /(3 \sqrt{2})=0.74 \cdots, a=0.5921, b=0.7072$, and $c$ $=0.601$. The entropy can be obtained by integrating this relation with respect to density as detailed in Ref. 53 where the integration constant is also reported. Values of $A$ have been taken from Table I of Ref. 52. They have been fitted to a polynomial to get

$$
\sqrt{A}=a_{1}\left(\phi_{\mathrm{fcc}}-\phi\right)+a_{2}\left(\phi_{\mathrm{fcc}}-\phi\right)^{2}+a_{3}\left(\phi_{\mathrm{fcc}}-\phi\right)^{3},
$$

with $a_{1}=0.35, a_{2}=-0.866$, and $a_{3}=3.58$. The final result for $n_{0}$ is reported in Fig. 3.

It is worth to note that the crystal phase is not commensurate, except at close packing. Therefore our results are consistent with the general statement by Prokof'ev and Svistunov: ${ }^{28}$ that commensurate crystals are not supersolids.

\section{Glass phase}

In order to understand the superglass phase, one must first translate the results of the classical hard sphere problem into the quantum one. Thus, let us start by discussing the classical results.

It is well known that the classical hard sphere problem may undergo a glass transition at a packing fraction $\phi_{K}$ $\sim 0.6$ if compressed sufficiently fast ${ }^{29,32-34,47}$ or in case of mixture of different particles. ${ }^{35,36}$ This has been found analytically under some approximations ${ }^{29,30,32-34}$ in simulations $^{35,54}$ and in experiments on colloidal systems. ${ }^{55,56}$ The classical phase diagram is reported in Fig. 2.

The physical mechanism behind this transition in finite dimension is still unclear. However, approximated theories able to provide good quantitative predictions have been developed. The most successful is the so-called mode-coupling theory (MCT) (Refs. 30, 47, and 57) that correctly describes the dynamics of the Langevin system [Eq. (1)] for densities slightly smaller than the glass transition density. A complementary approach is based on the replica method ${ }^{32-34,58}$ and gives predictions on static observables at and above the glass transition density. We will discuss in the following what predictions can be derived from these theories for the quantum problem.

Our strategy will consist in deriving results for the quantum glassy phase starting from the known results for the classical glassy phase. We will also provide quantitative results using the replica approach which has been shown to give a reasonable description of numerical simulation results. ${ }^{33,34}$ Our working hypothesis is that an ideal classical glass transition indeed takes place: at low density (or packing fraction) the system is in the liquid phase and above a critical density (assuming that crystallization is avoided) there is a thermodynamic transition toward an amorphous state of matter which is the glass state. In the glassy state the system can be frozen in many different amorphous configurations or states. Note that assuming that the classical glass transition is a real thermodynamic phase transition just simplifies the presentation. In fact, even if this is not the case in reality, one can translate and generalize all the following discussion: amorphous thermodynamic states will then become just metastable amorphous configurations in which the system is trapped on the relevant experimental time scales.

\section{Decomposition of the Gibbs measure in pure states}

Following the strategy outlined above, we will therefore assume that at the (classical) glass transition, the Gibbs mea- 
sure $P_{G}(\{\mathbf{x}\})=\left|\Psi_{G}(\{\mathbf{x}\})\right|^{2}$ splits into a very large number of thermodynamic states $P_{\alpha}(\{\mathbf{x}\})$,

$$
\left|\Psi_{G}(\{\mathbf{x}\})\right|^{2}=\sum_{\alpha} w_{\alpha} P_{\alpha}(\{\mathbf{x}\}) .
$$

An operative description of $P_{\alpha}(\{\mathbf{x}\})$ is the Boltzmann measure obtained by coupling the system to an infinitesimal nonhomogeneous external potential, $V_{\alpha}$, which forces the system into the state $\alpha$,

$$
P_{\alpha}(\{\mathbf{x}\})=\lim _{\epsilon \rightarrow 0} \frac{e^{-U_{N} / T-\epsilon V_{\alpha} / T}}{Z_{\alpha}}, \quad Z_{\alpha}=\lim _{\epsilon \rightarrow 0} \int d\{\mathbf{x}\} e^{-U_{N} / T-\epsilon V_{\alpha} / T} .
$$

Note that clearly the potential $V_{\alpha}$ must be chosen symmetric under particle exchanges. From the above definition it follows that the states $P_{\alpha}$ are also completely Bose symmetric like the full Boltzmann measure $P_{G}$.

The tricky aspect of glassy systems is that this external potential (or field) is not known a priori as it is for, say, the ferromagnetic case. The reason is that it is random as the state it pins. $w_{\alpha}$ is the thermodynamic weight of the state $\alpha$. It equals $Z_{\alpha} / Z_{N}$, where $Z_{\alpha}$ is the partition function in the presence of an infinitesimal pinning field forcing the system into the state $\alpha . Z_{N}$ is instead, as before, the partition function in the absence of any pinning field. Hence, it contains the contribution of all different states: $Z_{N}=\Sigma_{\alpha} Z_{\alpha}$ and then $\Sigma_{\alpha} w_{\alpha}=1$.

Since the $P_{\alpha}(\{\mathbf{x}\})$ s are equilibrium steady state distributions one can obtain an eigenfunction with minimum (zero) energy using the similarity transformation introduced in Eq. (9),

$$
\Psi_{\alpha}(\{\mathbf{x}\})=\mathcal{N}_{\alpha} \sqrt{Z_{N}} e^{U_{N} / 2 T} P_{\alpha}(\{\mathbf{x}\})=\mathcal{N}_{\alpha} P_{\alpha}(\{\mathbf{x}\}) / \Psi_{G}(\{\mathbf{x}\}) .
$$

$\mathcal{N}_{\alpha}$ is a normalization constant that has to be fixed by imposing that $\Psi_{\alpha}(\{\mathbf{x}\})$ is normalized,

$$
\begin{aligned}
1 & =\mathcal{N}_{\alpha}^{2} \lim _{\epsilon \rightarrow 0} \int d\{\mathbf{x}\} Z_{N} e^{U_{N} / T} \frac{e^{-2 U_{N} / T-2 \epsilon V_{\alpha} / T}}{Z_{\alpha}^{2}} \\
& =\mathcal{N}_{\alpha}^{2} \lim _{\epsilon \rightarrow 0} \frac{Z_{N}}{Z_{\alpha}^{2}} \int d\{\mathbf{x}\} e^{-U_{N} / T-2 \epsilon V_{\alpha} / T}=\mathcal{N}_{\alpha}^{2} \frac{Z_{N}}{Z_{\alpha}} .
\end{aligned}
$$

Note that we have used explicitly that one always recovers $Z_{\alpha}$ whether one uses $\epsilon$ or $2 \epsilon$. The outcome is that $\mathcal{N}_{\alpha}$ $=\sqrt{Z_{\alpha} / Z_{N}}=\sqrt{w_{\alpha}}$. Using this result and plugging expression (29) into Eq. (27) one finds

$$
\Psi_{G}(\{\mathbf{x}\})^{2}=\Psi_{G}(\{\mathbf{x}\}) \sum_{\alpha} \sqrt{w_{\alpha}} \Psi_{\alpha}(\{\mathbf{x}\})
$$

By factoring out $\Psi_{G}(\{\mathbf{x}\})$ one finally finds ${ }^{73}$

$$
\Psi_{G}(\{\mathbf{x}\})=\sum_{\alpha} \sqrt{w_{\alpha}} \Psi_{\alpha}(\{\mathbf{x}\})
$$

The ground-state wave function is therefore the coherent sum of the wave functions corresponding to states $\alpha$.

However, no interference is present in the thermodynamic limit and all cross terms in the square of the wave function can be dropped. This can be shown as follows: first, by plugging the expression of $\mathcal{N}_{\alpha}$ into Eq. (29) one finds that $\Psi_{\alpha}(\{\mathbf{x}\})=\sqrt{P_{\alpha}(\{\mathbf{x}\})}$. Using this result and noticing that the square of Eq. (30) has to give back Eq. (27) we find that the interference (cross-product) terms have to be zero. This result can be understood in a simple heuristic way: configurations on which the wave function $\Psi_{\alpha}$ is concentrated have extremely small weight in any other $\Psi_{\beta}$. Any given interference term $\alpha, \beta$ is expected to give a contribution decreasing exponentially fast in $N$. Since the number of states, hence the number of couples $\alpha, \beta$, increases at most exponentially in $N^{(d-1) / d}$ (see Ref. 59) one finds that the interference terms can be neglected in the thermodynamic limit.

Thus, the probability of finding the quantum system in a state $\alpha$ is not different from that of the classical problem: both are given by $w_{\alpha}$ in absence of a pinning external potential. The pinning potential $V_{\alpha}$ introduced in Eq. (28) induces a corresponding pinning potential $\mathcal{V}_{\alpha}$ via the mapping introduced in Eq. (8). The effect of the pinning potential is to concentrate the Jastrow wave function ${ }^{43}$ on state $\alpha$; equivalently, in the quantum problem, the pinning potential $\mathcal{V}_{\alpha}$ lifts the degeneracy between quantum ground states and selects $\Psi_{\alpha}$ as the unique ground state of the system. As a consequence each one of the $\Psi_{\alpha}$ s has to be interpreted as a possible state of the system (a more direct dynamical interpretation that shows that the system does not escape by tunneling once it is in a state $\alpha$ is presented in Sec. V). The glass phase in the quantum system is thus a random solid in the same sense as its classical counterpart. However, as much as in the case of the supercrystal, ODLRO can still develop, leading to a nonvanishing condensate fraction, which we will study below.

Translating the classical results for hard spheres ${ }^{33}$ in the quantum case we thus find that the quantum ground state is unique for $\phi<\phi_{K}$ and is degenerate for $\phi>\phi_{K}$ but the logarithm of the number of ground states is subextensive and therefore the entropy remains zero above $\phi_{K}$. It follows that the phase transition is not manifested by a nonanalyticity in the free energy of the system. However, suitable correlation functions (such as the "point to set" or "dynamical" correlation functions ${ }^{60,61}$ ) should display a growing correlation length at the transition. Also the structure function $g(r)$ shows a (weak) discontinuity at $\phi_{K}$, but it does not display any long-range order. In the following we will study the properties of this glassy quantum state, in particular the ones related to ODLRO.

\section{Condensate fraction and corresponding Edwards-Anderson parameter}

Using the results above on the ground-state wave function one can readily obtain the one-particle off-diagonal density matrix. As interference terms can be neglected, it reads

$$
\mathcal{R}\left(\mathbf{x}, \mathbf{x}^{\prime}\right)=\sum_{\alpha} w_{\alpha} \mathcal{R}_{\alpha}\left(\mathbf{x}, \mathbf{x}^{\prime}\right)=V \sum_{\alpha} w_{\alpha} \sum_{i} n_{i, \alpha} \psi_{i, \alpha}(\mathbf{x}) \psi_{i, \alpha}^{*}\left(\mathbf{x}^{\prime}\right),
$$

where the $n_{i, \alpha}$ and the $\psi_{i, \alpha}(\mathbf{x})$ are, respectively, eigenvalues and eigenvectors of the one-particle density matrix 
$\mathcal{R}_{\alpha}\left(\mathbf{x}, \mathbf{x}^{\prime}\right)$. (Because the wave functions $\Psi_{\alpha}$ are real, the $\mathcal{R}_{\alpha}\left(\mathbf{x}, \mathbf{x}^{\prime}\right)$ are real and symmetric, and the eigenvalues and eigenvectors are real.) The $n_{i, \alpha}$ have a physical interpretation: they are the fraction of particles in the quantum state $i$ within the thermodynamic state $\alpha$, and $\Sigma_{\alpha, i} w_{\alpha} n_{i, \alpha}=1$ accounts for all the particles in all possible states $\alpha$ with probabilities $w_{\alpha}$.

For large separations $\left|\mathbf{x}-\mathbf{x}^{\prime}\right| \rightarrow \infty$, one has for each $\mathcal{R}_{\alpha}$,

$\lim _{\left|\mathbf{x}-\mathbf{x}^{\prime}\right| \rightarrow \infty} \mathcal{R}_{\alpha}\left(\mathbf{x}, \mathbf{x}^{\prime}\right)=f_{\alpha}(\rho) \frac{\rho_{\alpha}(\mathbf{x}) \rho_{\alpha}\left(\mathbf{x}^{\prime}\right)}{\rho^{2}}=n_{0, \alpha} V \psi_{0, \alpha}(\mathbf{x}) \psi_{0, \alpha}^{*}\left(\mathbf{x}^{\prime}\right)$.

Proceeding similarly to the case of the crystal, we obtain $\psi_{0, \alpha}(\mathbf{x})=\sqrt{\frac{f_{\alpha}(\rho)}{n_{0, \alpha} V}} \rho_{\alpha}(\mathbf{x}) / \rho$. We use the normalization of the eigenvector $\psi_{0, \alpha}$ to write

$$
n_{0, \alpha}=f_{\alpha}(\rho) \frac{1}{\rho^{2}} \frac{1}{V} \int d \mathbf{x} \rho_{\alpha}(\mathbf{x})^{2},
$$

and for the total condensate fraction we get

$$
n_{0}=\frac{1}{\rho^{2}} \sum_{\alpha} w_{\alpha} f_{\alpha}(\rho) \frac{1}{V} \int d \mathbf{x} \rho_{\alpha}(\mathbf{x})^{2}
$$

We expect that the $f_{\alpha}(\rho)$ 's are independent of the state $\alpha$ since they are thermodynamic quantities related to the addition of one single particle to the system [recall Eq. (16)]. In order to quantify the inhomogeneity of the glass state one can introduce the correlation function of the density and condensate wave function fluctuations that read, respectively,

$$
\begin{gathered}
\widetilde{g}\left(\mathbf{x}, \mathbf{x}^{\prime}\right) \equiv \rho^{-2} \sum_{\alpha} w_{\alpha} \rho_{\alpha}(\mathbf{x}) \rho_{\alpha}\left(\mathbf{x}^{\prime}\right), \\
G_{\psi}\left(\mathbf{x}, \mathbf{x}^{\prime}\right) \equiv \sum_{\alpha} w_{\alpha} \psi_{0, \alpha}(\mathbf{x}) \psi_{0, \alpha}\left(\mathbf{x}^{\prime}\right) .
\end{gathered}
$$

Actually, using Eq. (32) we find that they are related one to the other by a simple proportionality relation,

$$
\widetilde{g}\left(\mathbf{x}, \mathbf{x}^{\prime}\right)=\frac{n_{0} V}{f(\rho)} \sum_{\alpha} w_{\alpha} \psi_{0, \alpha}(\mathbf{x}) \psi_{0, \alpha}\left(\mathbf{x}^{\prime}\right) \equiv \frac{n_{0} V}{f(\rho)} G_{\psi}\left(\mathbf{x}, \mathbf{x}^{\prime}\right) .
$$

Substituting these definitions in Eq. (34) we get

$$
\begin{aligned}
n_{0} & =f(\rho) \frac{1}{V} \int d \mathbf{x} \frac{1}{\rho^{2}} \sum_{\alpha} w_{\alpha} \rho_{\alpha}(\mathbf{x})^{2} \\
& =f(\rho) \frac{1}{V} \int d \mathbf{x} \widetilde{g}(\mathbf{x}, \mathbf{x})=f(\rho) \widetilde{g}(0),
\end{aligned}
$$

where we used that $\widetilde{g}\left(\mathbf{x}, \mathbf{x}^{\prime}\right)=\widetilde{g}\left(\left|\mathbf{x}-\mathbf{x}^{\prime}\right|\right)=\widetilde{g}(r)$, as translational invariance is restored after averaging over all possible states. Note that $\widetilde{g}\left(\mathbf{x}, \mathbf{x}^{\prime}\right)$ plays the role of an EdwardsAnderson order parameter for the glass state. For instance, one can define

$$
q_{\mathrm{EA}}=\sum_{\alpha} w_{\alpha} \frac{1}{V} \int d \mathbf{x}\left(\frac{\rho_{\alpha}(\mathbf{x})}{\rho}-1\right)^{2}=\widetilde{g}(0)-1 .
$$

Since the density and condensate wave function fluctuations are proportional $\widetilde{g}\left(\mathbf{x}, \mathbf{x}^{\prime}\right)$ also represents (up to a proportionality constant) the inhomogeneity of the condensate wave function. It is a quantitative measure of how much the condensate is amorphous. It can be computed in numerical simulation. Look, for example, to Fig. 3 of Ref. 20 which provides a visual representation of the inhomogeneity captured by $\widetilde{g}\left(\mathbf{x}, \mathbf{x}^{\prime}\right)$.

In order to obtain quantitative results on the condensate fraction we have to compute $\widetilde{g}(0)$ and $f(\rho)$. The replica method, ${ }^{32}$ in particular the small cage expansion, ${ }^{58}$ has been successfully applied to describe the glassy phase of hard spheres. $^{33,34}$ These two quantities can be indeed obtained using the procedure detailed in Refs. 33 and 34. Since this is well documented ${ }^{74}$ we do not reproduce the computation and just quote the final result which is very similar to the one for the crystal,

$$
n_{0}=\frac{f(\rho)}{\rho} \frac{1}{(4 \pi A)^{3 / 2}},
$$

where $A$ is the so-called cage radius and is a measure of particle vibrations. Hence, by using the equation of state for the glass and the values of $A$ reported in Refs. 33 and 34 we finally obtain a quantitative result for the glass condensate fraction which is reported in Fig. 3. Note that the results of Refs. 33 and 34 depend slightly on the approximation that is used to describe the liquid; for consistency, we used the results obtained using the Carnahan-Starling approximation as we did for the liquid.

The replica method allows us to compute both $g(r)$ and $\widetilde{g}(r)$ in the glass phase but within a different approximation scheme known as HNC (hypernetted chain) approximation. ${ }^{50}$ The results, taken from Refs. 32 and 33, are reproduced in Fig. 4.

\section{QUANTUM SLOW DYNAMICS AND THE APPROACH TO THE QUANTUM GLASS TRANSITION}

In the following we shall focus on the real-time dynamics of the superfluid phase when the transition to the superglass is approached. Also in this case, the knowledge of dynamical correlation functions for Brownian hard spheres will allow us to obtain results on dynamical correlation functions of the corresponding quantum problem. In Sec. V A we will explain how the mapping works for dynamic observables.

\section{A. Mapping from Brownian dynamics of hard spheres to real-time dynamics of the quantum model}

In order to show how one can obtain information about the real-time quantum dynamics from Langevin dynamics (1) it is useful to introduce a bra-ket notation for the FokkerPlanck problem; we define $P(\{\mathbf{x}\}, t)=\langle\{\mathbf{x}\} \mid P(t)\rangle$, and we denote by $|G\rangle$ the Gibbs distribution (5), $P_{G}(\{\mathbf{x}\}, t)=\langle\{\mathbf{x}\} \mid G\rangle$ $=\exp \left[-U_{N}(\{\mathbf{x}\}) / T\right] / Z_{N}$, such that $H_{\mathrm{FP}}|G\rangle=0$. We also denote by $\langle+|$ the constant state, $\langle+\mid\{\mathbf{x}\}\rangle=1$. Note that 

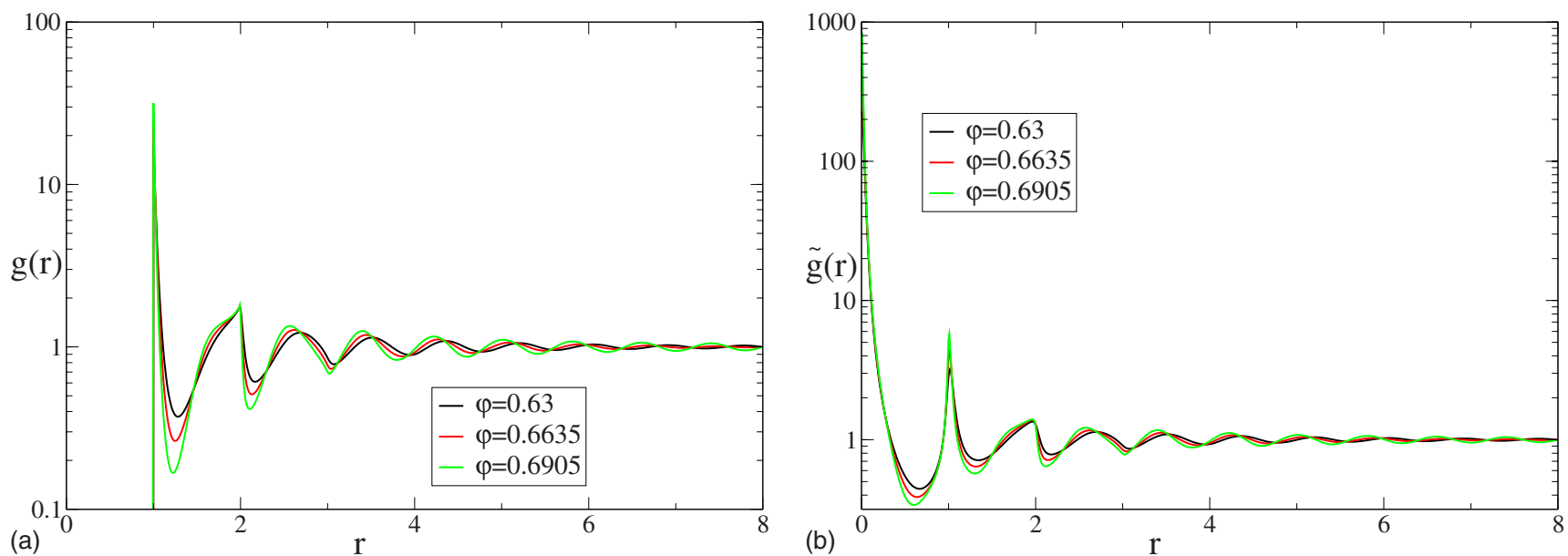

FIG. 4. (Color online) Correlation function of the density (left) and of the condensate wave function (right) for the quantum system corresponding to classical hard spheres at different densities in the glass phase. Details of the computations are in Ref. 33. Note that these quantities can be measured in quantum Monte Carlo numerical simulations (see, e.g., Ref. 20).

$$
H_{\mathrm{FP}}^{\dagger}=e^{U_{N} / T} H_{\mathrm{FP}} e^{-U_{N} / T},
$$

which is consistent with Eq. (6) and the fact that $H$ is Hermitian. Then

$$
\begin{aligned}
H_{\mathrm{FP}}|G\rangle & =0 \Rightarrow 0=\langle G| H_{\mathrm{FP}}^{\dagger}=\langle G| e^{U_{N} / T} H_{\mathrm{FP}} e^{-U_{N} / T} \\
& =Z_{N}^{-1}\langle+| H_{\mathrm{FP}} e^{-U_{N} / T} \Rightarrow 0=\langle+| H_{\mathrm{FP}},
\end{aligned}
$$

i.e., $\langle+|$ is a left eigenvector of $H_{\mathrm{FP}}$ with zero eigenvalue.

One observable that is particularly interesting to characterize the quantum dynamics is the dynamical structure factor, $F_{Q}(q, t)$, which is the time-dependent correlation function of a Fourier component of the density operator, $\rho_{q}(\{\mathbf{x}\})=\Sigma_{l} e^{i \mathbf{q} \cdot \mathbf{x}_{\mathbf{1}}}$. We shall show that the dynamical structure factor for the quantum problem is related to the imaginary time analytic continuation of the dynamical structure factor, $F_{c l}(q, t)$, for Brownian hard spheres.

Observing that $\left\langle\{\mathbf{x}\}\left|e^{-t H_{\mathrm{FP}}}\right|\{\mathbf{y}\}\right\rangle$ is the probability of going from $\{\mathbf{y}\}$ to $\{\mathbf{x}\}$ in time $t$, we can write the Brownian correlation function as follows:

$$
\begin{aligned}
F_{\mathrm{cl}}(q, t)= & \left\langle\rho_{q}(t) \rho_{-q}(0)\right\rangle \\
\equiv & \int d\{\mathbf{x}\} d\{\mathbf{y}\} \rho_{q}(\{\mathbf{x}\}) \\
& \times\left\langle\{\mathbf{x}\}\left|e^{-t H_{\mathrm{FP}}}\right|\{\mathbf{y}\}\right\rangle \rho_{-q}(\{\mathbf{y}\}) \frac{e^{-\beta U_{N}(\{\mathbf{y}\})}}{Z_{N}} \\
= & \left\langle+\left|\rho_{q} e^{-t H_{\mathrm{FP}}} \rho_{-q}\right| G\right\rangle \\
= & \langle+| e^{t H_{\mathrm{FP}}} \rho_{q} e^{-t H_{\mathrm{FP}} \rho_{-q}|G\rangle} \\
= & \left\langle+\left|e^{-U_{N} /(2 T)} e^{t H} \rho_{q} e^{-t H} e^{U_{N} /(2 T)} \rho_{-q}\right| G\right\rangle \\
= & \left\langle 0\left|e^{t H} \rho_{q} e^{-t H} \rho_{-q}\right| 0\right\rangle=\sum_{n}\left|\left\langle 0\left|\rho_{q}\right| n\right\rangle\right|^{2} e^{-t\left(E_{n}-E_{0}\right)} \\
= & \int_{0}^{\infty} \frac{d \omega}{2 \pi} \rho_{q}(\omega) e^{-\omega t},
\end{aligned}
$$

where $|0\rangle \equiv \sqrt{Z_{N}} e^{U_{N} /(2 T)}|G\rangle$ is quantum ground state (10), $|n\rangle$ are the excited states, and

$$
\rho_{q}(\omega) \equiv 2 \pi \sum_{n}\left|\left\langle 0\left|\rho_{q}\right| n\right\rangle\right|^{2} \delta\left(E_{n}-E_{0}-\omega\right)
$$

is the distribution of classical (inverse) relaxation times for the density fluctuations.

We are interested in the quantum correlations; the quantum response function is given by ${ }^{62}$

$$
\begin{aligned}
R_{Q}(q, t) & =i \theta(t)\left\langle 0\left|\left[e^{i t H} \rho_{q} e^{-i t H}, \rho_{-q}\right]\right| 0\right\rangle \\
& =i \theta(t) \sum_{n}\left|\left\langle 0\left|\rho_{q}\right| n\right\rangle\right|^{2}\left[e^{-i t\left(E_{n}-E_{0}\right)}-e^{i t\left(E_{n}-E_{0}\right)}\right] \\
& =-2 \theta(t) \int_{0}^{\infty} \frac{d \omega}{2 \pi} \rho_{q}(\omega) \sin (\omega t) .
\end{aligned}
$$

It follows that the imaginary part of its Fourier transform reads

$$
R_{Q}^{\prime \prime}(q, \omega)=\frac{1}{2} \operatorname{sgn}(\omega) \rho_{q}(|\omega|)
$$

and using the quantum (bosonic) fluctuation-dissipation theorem at zero temperature, we get the quantum correlation function,

$$
\begin{aligned}
S_{Q}(q, \omega) & =\frac{1}{2} \operatorname{sgn}(\omega) R_{Q}^{\prime \prime}(q, \omega) \\
& =\frac{1}{2} \rho_{q}(|\omega|) \Rightarrow F_{Q}(q, t)=\int_{-\infty}^{\infty} \frac{d \omega}{2 \pi} S_{Q}(q, \omega) e^{-i \omega t} \\
& =\int_{0}^{\infty} \frac{d \omega}{2 \pi} \rho_{q}(\omega) \cos (\omega t)
\end{aligned}
$$

This final simple expression allows us to obtain results on the dynamical structure factor starting from the distribution of (inverse) relaxation times.

\section{B. Results for the time-dependent density-density correlator}

Assume that the density correlator has been normalized in such a way that $F(q, t=0)=1$, then $\int_{0}^{\infty} \frac{d \omega}{2 \pi} \rho_{q}(\omega)=1$. In the 

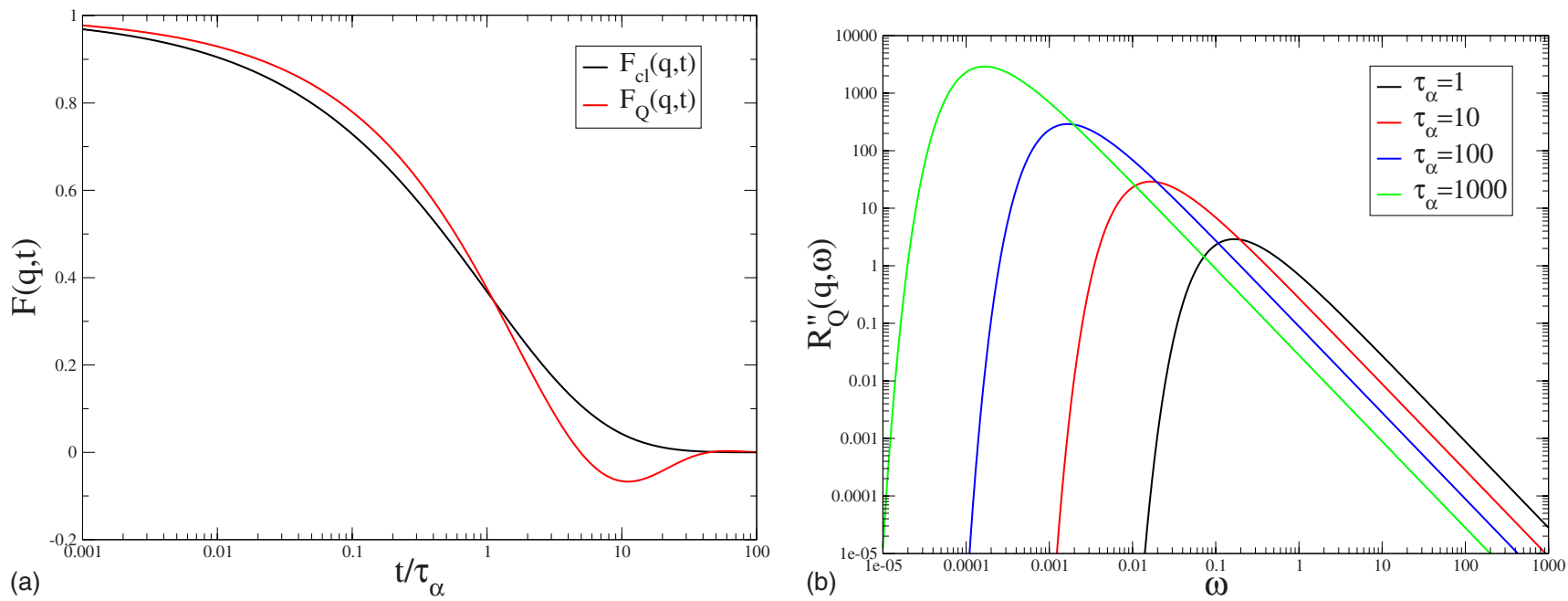

FIG. 5. (Color online) (Left) Long-time shape of the correlation function [Eq. (49)] $F_{\mathrm{cl}}(q, t)$ $\sim \exp \left(-\sqrt{t / \tau_{\alpha}}\right)$ and $F_{Q}(q, t) \sim \exp \left(-\sqrt{t / 2 \tau_{\alpha}}\right) \cos \left(\sqrt{t / 2 \tau_{\alpha}}\right)$ as a function of $t / \tau_{\alpha}$ in a linear-logarithmic scale. (Right) Imaginary part of the response function of the quantum problem [Eqs. (48) and (49)] as a function of frequency for different values of $\tau_{\alpha}$ (in arbitrary units).

glass transition literature, different phenomenological expressions have been used in order to describe the slowing down of the relaxation of $F(q, t)$ on approaching the glass transition.

In dielectric spectroscopy one often writes

$$
F_{\mathrm{cl}}(q, t)=\int_{-\infty}^{\infty} d \ln \tau G_{q}(\ln \tau) e^{-t / \tau}, \quad G_{q}(\ln \tau)=\frac{\rho_{q}(1 / \tau)}{2 \pi \tau},
$$

and to describe the slow relaxation (low-frequency) part it is assumed that $G_{q}(\ln \tau)$ contains a single time scale $\tau_{\alpha}(q)$, $G_{q}(\ln \tau)=\left[\tau / \tau_{\alpha}(q)\right] g\left[\tau / \tau_{\alpha}(q)\right]$. The scaling function $g(x)$ might depend weakly on $q$. Then

$$
\begin{gathered}
F_{\mathrm{cl}}(q, t)=\int_{0}^{\infty} d x g(x) e^{-t /\left[x \tau_{\alpha}(q)\right]}=f_{\mathrm{cl}}\left[t / \tau_{\alpha}(q)\right], \\
F_{Q}(q, t)=\int_{-\infty}^{\infty} d \ln \tau G_{q}(\ln \tau) \cos (t / \tau) \\
=\int_{0}^{\infty} d x g(x) \cos \left(\frac{t}{x \tau_{\alpha}(q)}\right)=f_{Q}\left[t / \tau_{\alpha}(q)\right], \\
R_{Q}^{\prime \prime}(q, \omega)=\frac{\pi}{\omega} \frac{1}{\omega \tau_{\alpha}(q)} g\left(\frac{1}{\omega \tau_{\alpha}(q)}\right) .
\end{gathered}
$$

Several forms have been used successfully in the literature (see Ref. 63 for a review).

(1) Stretched exponential $\beta=1 / 2$. The simplest nontrivial case is $f_{\mathrm{cl}}(y)=e^{-\sqrt{y}}$; in this case

$$
g(x)=\frac{e^{-x / 4}}{\sqrt{4 \pi x}} \Rightarrow f_{Q}(y)=e^{-\sqrt{y / 2}} \cos [\sqrt{y / 2}] .
$$

(2) Stretched exponential. For $f_{\mathrm{cl}}(y)=e^{-y^{\beta}}$ and generic $\beta$ we have

$$
g(x)=\frac{1}{\pi x} \int_{0}^{\infty} d s e^{-s-(x s)^{\beta} \cos (\pi \beta)} \sin \left[(x s)^{\beta} \sin (\pi \beta)\right],
$$

and in this case $f_{Q}(y)$ must be computed numerically.

(3) Cole-Davidson. Another common expression corresponding to

$$
g(x)=\frac{\sin (\pi \gamma)}{\pi}\left(\frac{x}{1-x}\right)^{\gamma} \quad 0 \leq x \leq 1
$$

In this case $f_{Q}(y)$ can be computed in terms of hypergeometric functions.

Here we limit ourselves to illustrate the qualitative behavior of $F_{Q}(q, t)$ on approaching the glass transition. For this, we consider solely the first case. (We checked numerically that the other forms give qualitatively similar resultsoscillations in $F_{Q}$ are much more pronounced if one uses the Cole-Davidson form due to the frequency cutoff.) Some examples constructed using Eq. (49) are reported in Fig. 5. The classical correlation function decays from 1 to 0 over the time scale $\tau_{\alpha}$; the corresponding (real-time) quantum correlation function, directly accessible in numerical simulations, displays the same slow decay modulated by oscillations according to Eq. (49). The imaginary part of the response function, directly accessible in experiments, shows a peak at $\omega$ $\sim 1 / \tau_{\alpha}$, whose amplitude increases $\propto \tau_{\alpha}$ upon increasing $\tau_{\alpha}$. Note that usually in classical glassy systems these lowfrequency features are accompanied by faster relaxations, related to "intracage" motion and/or fast molecular relaxations. These have been neglected here and in Fig. 5 but we expect them to be present in the quantum case as well. They would appear as secondary peaks at higher frequency, and typically their density dependence is weak. The complete time dependence of the density-density correlation function would therefore be a first rapid relaxation to a plateau value and then a second one which is the one studied in detail in this section. At the glass transition this second relaxation does not take place anymore since the relaxation time diverges (or 
it is larger than any experimental time scale). As a consequence part of the density fluctuations becomes frozen. The plateau value in the correlation function measures precisely that. Actually, the plateau value for a wave vector $q$ will coincide with the Fourier transform of the function $\widetilde{g}(\mathbf{x}$ $-\mathbf{x}^{\prime}$ ) defined in Eq. (35).

\section{Condensate fluctuations}

To conclude this section we will focus on the dynamical fluctuations of the condensate wave function. As we already discussed, in the glass phase the system can be in many different states, each one characterized by a different density profile and by a condensate wave function, $\psi_{0, \alpha}(\mathbf{x})$, which in our model is simply proportional to the classical density profile of the corresponding state. In Eq. (36) we defined the correlation function of the fluctuations of the condensate wave function when the system is frozen in a given amorphous state. This has the form reported in Fig. 4 and can be accessed by a direct computation of the condensate wave function as done in Ref. 20. It is important to notice that it can also be obtained from purely dynamical measurements as we will now show.

Consider the time-dependent one-particle density matrix; in second quantization it is defined as $\mathcal{R}_{Q}\left(t ; \mathbf{x}, \mathbf{x}^{\prime}\right)$ $\equiv\left\langle\hat{\psi}^{\dagger}(\mathbf{x}, t) \hat{\psi}\left(\mathbf{x}^{\prime}, 0\right)\right\rangle$, where $\hat{\psi}(\mathbf{x}, t)$ is the standard bosonic annihilation operator. Within first quantization, it can be written as follows:

$$
\begin{aligned}
\mathcal{R}_{Q}\left(t ; \mathbf{x}, \mathbf{x}^{\prime}\right)= & V \int d \mathbf{x}_{2} \cdots d \mathbf{x}_{N} d \mathbf{x}_{2}^{\prime} \cdots d \mathbf{x}_{N}^{\prime} \Psi_{G}\left(\mathbf{x}, \mathbf{x}_{2}, \ldots, \mathbf{x}_{N}\right) \\
& \times\left\langle\mathbf{x}_{2}, \ldots, \mathbf{x}_{N}\left|e^{-i t H}\right| \mathbf{x}_{2}^{\prime}, \ldots, \mathbf{x}_{N}^{\prime}\right\rangle \\
& \times \Psi_{G}\left(\mathbf{x}^{\prime}, \mathbf{x}_{2}^{\prime}, \ldots, \mathbf{x}_{N}^{\prime}\right) .
\end{aligned}
$$

Clearly for $t=0$ Eq. (52) gives back Eq. (14). In order to understand the time-dependent behavior it is useful to consider the evolution in imaginary time and use again the mapping on the Langevin dynamics. From Eq. (6) we have

$$
\begin{aligned}
\left\langle\mathbf{x}_{2}, \ldots, \mathbf{x}_{N}\left|e^{-t H}\right| \mathbf{x}_{2}^{\prime}, \ldots, \mathbf{x}_{N}^{\prime}\right\rangle \\
=\left[\Psi_{G}\left(\mathbf{x}_{2}, \ldots, \mathbf{x}_{N}\right)\right]^{-1}\left\langle\mathbf{x}_{2}, \ldots, \mathbf{x}_{N}\left|e^{-t H_{\mathrm{FP}}}\right| \mathbf{x}_{2}^{\prime}, \ldots, \mathbf{x}_{N}^{\prime}\right\rangle \\
\quad \times \Psi_{G}\left(\mathbf{x}_{2}^{\prime}, \ldots, \mathbf{x}_{N}^{\prime}\right),
\end{aligned}
$$

where $\Psi_{G}\left(\mathbf{x}_{2}, \ldots, \mathbf{x}_{N}\right)$ is Jastrow state (10) (Ref. 43) for $N$ -1 particles. Plugging Eq. (53) in Eq. (52) and using explicit form (10), we finally obtain

$$
\begin{aligned}
\mathcal{R}\left(t ; \mathbf{x}, \mathbf{x}^{\prime}\right)= & V \int d \mathbf{x}_{2} \cdots d \mathbf{x}_{N} d \mathbf{x}_{2}^{\prime} \cdots d \mathbf{x}_{N}^{\prime} \\
& \times \exp \left(-\frac{1}{2} \sum_{i=2}^{N} V\left(\mathbf{x}-\mathbf{x}_{i}\right)\right) \\
& \times\left\langle\mathbf{x}_{2}, \ldots, \mathbf{x}_{N}\right| e^{-t H_{\mathrm{FP}}\left|\mathbf{x}_{2}^{\prime}, \ldots, \mathbf{x}_{N}^{\prime}\right\rangle} \\
& \times \exp \left[-\frac{1}{2} \sum_{i=2}^{N} V\left(\mathbf{x}^{\prime}-\mathbf{x}_{i}^{\prime}\right)\right] \frac{e^{-U_{N-1}\left(\mathbf{x}_{2}^{\prime}, \ldots, \mathbf{x}_{N}^{\prime}\right)}}{Z_{N}},
\end{aligned}
$$

where $U_{N-1}$ is the interaction potential of the $N-1$ particles and we have used the notation $\mathcal{R}$ for the imaginary time continuation of $\mathcal{R}_{Q}$.

In the special case of a Jastrow hard sphere wave function, ${ }^{43}$ the factors of $\frac{1}{2}$ are irrelevant. Then Eq. (54) becomes

$$
\begin{aligned}
\mathcal{R}\left(t ; \mathbf{x}, \mathbf{x}^{\prime}\right)= & V \int d \mathbf{x}_{2} \cdots d \mathbf{x}_{N} d \mathbf{x}_{2}^{\prime} \cdots d \mathbf{x}_{N}^{\prime} \exp \left[-\sum_{i=2}^{N} V\left(\mathbf{x}-\mathbf{x}_{i}\right)\right] \\
& \times\left\langle\mathbf{x}_{2}, \ldots, \mathbf{x}_{N}\left|e^{-t H_{\mathrm{FP}}}\right| \mathbf{x}_{2}^{\prime}, \ldots, \mathbf{x}_{N}^{\prime}\right\rangle \frac{e^{-U_{N}\left(\mathbf{x}^{\prime}, \mathbf{x}_{2}^{\prime}, \ldots, \mathbf{x}_{N}^{\prime}\right)}}{Z_{N}}
\end{aligned}
$$

and has a straightforward interpretation in terms of Brownian dynamics of hard spheres: one should pick up a configuration $\left\{\mathbf{x}^{\prime}\right\}$ from the equilibrium distribution, such that particle 1 is in $\mathbf{x}_{1}^{\prime}=\mathbf{x}^{\prime}$. Then particle 1 must be removed and particles $2, \ldots, N$ evolved according to the Langevin dynamics in absence of particle 1 . Finally, one should attempt to reintroduce particle 1 at position $\mathbf{x}_{1}=\mathbf{x}$. The function $\mathcal{R}\left(t ; \mathbf{x}, \mathbf{x}^{\prime}\right)$ is the probability that the attempt is successful or in other words that there is a void at time $t$ around $\mathbf{x}$ large enough to allow the reinsertion of particle 1 .

Close to the glass transition, as we already discussed, there is a huge separation of time scales in the classical dynamics between a "fast" intrastate relaxation and a "slow" relaxation corresponding to hopping between different states and characterized by a growing time scale $\tau_{\alpha}$. If we remove particle 1 at $t=0$, the perturbation of the density field will not relax inside the initial state until $t \sim \tau_{\alpha}$, where the state will change. This implies that $\mathcal{R}\left(t ; \mathbf{x}, \mathbf{x}^{\prime}\right)$ will have a plateau at times $\tau_{\text {fast }} \ll t \lesssim \tau_{\alpha}$ corresponding to the stationary probability inside the initial state at $t=0$. This is given by

$$
\mathcal{R}\left(\tau_{\text {fast }} \ll t \lesssim \tau_{\alpha} ; \mathbf{x}, \mathbf{x}^{\prime}\right) \propto \sum_{\alpha} w_{\alpha} \psi_{0, \alpha}(\mathbf{x}) \psi_{0, \alpha}\left(\mathbf{x}^{\prime}\right) \propto \widetilde{g}\left(\mathbf{x}-\mathbf{x}^{\prime}\right),
$$

where one has to average over all possible initial states. As obtained in Sec. V B for the density-density correlation function, we expect that in presence of a huge separation of time scales, as it is the case close to the glass transition (or in the glass state where the second relaxation does not take place anymore $)$, one finds that $\mathcal{R}\left(\tau_{\text {fast }} \ll t \lesssim \tau_{\alpha} ; \mathbf{x}, \mathbf{x}^{\prime}\right) \sim \mathcal{R}_{Q}\left(\tau_{\text {fast }}\right.$ $\left.\ll t \lesssim \tau_{\alpha} ; \mathbf{x}, \mathbf{x}^{\prime}\right)$. One way to understand this result consists in expressing $\mathcal{R}(t)$ in terms of the Fourier transform of $\mathcal{R}_{Q}$ : $\mathcal{R}(t)=\int \frac{d \omega}{2 \pi} \mathcal{R}_{Q}(\omega) \exp (-\omega t)$. If $\mathcal{R}$ displays a very long plateau this means that $\mathcal{R}_{Q}(\omega)$ contains two distinct contributions corresponding to $\omega \propto 1 / \tau_{\text {fast }}$ and $\omega \propto 1 / \tau_{\alpha}$. As a consequence, $\mathcal{R}_{Q}$ on times intermediate between fast and slow time scales will coincide with $\mathcal{R}$ since the contribution from large frequencies (of the order or $1 / \tau_{\text {fast }}$ ) will have died out and the contribution from the very long frequencies (of the order of $\left.1 / \tau_{\alpha}\right)$ will be the same since $\exp (-i \omega t) \simeq \exp (-\omega t) \simeq 1$. As a conclusion, $\mathcal{R}_{Q}$ will display a plateau whose extension will become infinite beyond the glass transition. The value of $\mathcal{R}_{Q}$ on the plateau corresponds to the fraction of frozen condensate wave function fluctuations and equals $G_{\psi}$ (and hence is 
proportional to $\widetilde{g}$ ). Therefore this quantity can also be computed in a dynamic framework without introducing replicas.

\section{SUPERFLUID PROPERTIES AND PHASE DIAGRAM OF REALISTIC SUPERGLASS PHASES}

The conclusion from Secs. IV and V is that the ground state of the model can be a liquid, a crystal, or a glass and all these phases are characterized by a finite condensate fraction $n_{0}$. However, the study of the superfluid properties requires also the knowledge of excited states or at least of the excitation spectrum. The latter is related to superfluid properties by the celebrated Landau argument ${ }^{64}$ that predicts for the critical velocity,

$$
v_{c} \leq \min _{k}[\epsilon(k) / k]
$$

In the zero-temperature liquid $\mathrm{He}^{4}$ phase, the excitation spectrum is linear at small $k$ and has a minimum at larger $k$; therefore $v_{c}$ is finite and the system is superfluid.

Unfortunately, for the Jastrow wave function ${ }^{43}$ discussed above [Eq. (10)], one can show that the excitation spectrum is quadratic at small $k$ at least if the potential $V(\mathbf{x})$ has finite integral. Therefore Eq. (57) implies that $v_{c}=0$ and the system is not superfluid, much as it happens for an ideal Bose gas despite the condensate fraction equals 1 at zero temperature.

The quadratic spectrum of Jastrow wave functions ${ }^{43}$ can be related to the following properties of Hamiltonian (7):

(1) The ground-state energy per particle $e(\rho)$ of the Jastrow ground state ${ }^{43}$ is always zero, therefore the pressure $P=\rho^{2} \frac{d e}{d \rho}=0$ and the compressibility is infinite, $\chi_{T}^{-1}=\rho \frac{d P}{d \rho}=0$.

(2) Consequently the sound velocity $c=1 / \sqrt{\rho \chi_{T}}=0$, i.e., there are no phonons and the linear part of the spectrum at small $k$ is absent; this is because no restoring force for density fluctuations is present if $e(\rho)$ is independent of $\rho$.

(3) The static structure factor of the Jastrow wave function ${ }^{43}$ has the property $\lim _{k \rightarrow 0} S(k) \neq 0$; therefore, the Feynman relation ${ }^{68} \epsilon(k)=k^{2} /[2 m S(k)]$ gives $\epsilon(k) \propto k^{2}$, consistent with $c=0$.

(4) Finally, it is possible to identify a hidden symmetry in the problem, related to the special form of the potential in Eq. (7), which is responsible for nontrivial cancellations in the Bogoliubov low-density perturbation theory for Eq. (7) around the ideal gas limit. Again these cancellations are responsible for the absence of the linear part of the spectrum.

When using Jastrow wave functions ${ }^{43}$ as variational functions for liquid helium, a classical strategy ${ }^{45}$ to reintroduce phonons is to add a nonintegrable part to the Jastrow potential, ${ }^{43}$ such that $V(\mathbf{x}) \sim|\mathbf{x}|^{-2}$ at large $|\mathbf{x}|$. In this way $S(k) \sim k$ at small $k$ and the excitation spectrum is linear. This is also quite natural since long-range correlations are expected in the ground state of generic quantum systems even if the original interaction is short ranged. The inclusion of these terms in our formalism is possible and their presence does not influence much the results for the properties of the glass, e.g., for $g(r)$ and $\widetilde{g}(r)$ discussed in Sec. IV D, except at large $r$ or small $k$.

However, the classical-quantum mapping will give in this case quantum Hamiltonian (7) with very long ranged inter- action, while we would like to keep the original local nature of the quantum Hamiltonian. Therefore we consider an alternative way to solve the problem: we propose to introduce a perturbation of quantum Hamiltonian (7) by adding a small potential term $\Delta \mathcal{V}_{N}(\{\mathbf{x}\})=\sum_{i<j} \delta u\left(\mathbf{x}_{i}-\mathbf{x}_{j}\right)$ and treat it in perturbation theory.

The situation here is quite similar to Bogoliubov lowdensity perturbation theory: ${ }^{65}$ indeed, the ideal Bose gas has a finite condensate fraction (actually equals 1) but is not superfluid because its excitation spectrum is quadratic. Once an infinitesimal interaction is added (or in the very dilute regime), the spectrum immediately becomes linear and the system becomes superfluid. ${ }^{65}$ Hence by analogy we argue that perturbation theory can be applied in our case. As a check of this argument we verified that in the low-density limit, as discussed above, the Bogoliubov theory of the unperturbed model leads to a quadratic spectrum due to a hidden symmetry. On the contrary, the perturbation $\Delta \mathcal{V}_{N}$ breaks the hidden symmetry, and in its presence the usual Bogoliubov theory applies and leads to a linear spectrum.

Using perturbation theory at first order, it is straightforward to show that

$$
e(\rho)=\frac{\rho}{2} \int \operatorname{drg}(r) \delta u(r),
$$

where $g(r)$ is the correlation function of the hard sphere liquid at density $\rho$. One can show by explicit computation for a suitable specific form of $\delta u(r)$ that, at the glass transition density $\rho_{K}$, the ground-state energy is continuous but its first derivative has a jump.

The pressure $P(\mu)$ (as a function of the chemical potential $\mu)$ is the Legendre transform of $e(\rho)$,

$$
P(\mu)=\max _{\rho}[\rho \mu-\rho e(\rho)],
$$

therefore

$$
\begin{aligned}
& \mu(\rho)=\frac{d}{d \rho}[\rho e(\rho)], \\
& P(\rho)=\rho^{2} \frac{d}{d \rho} e(\rho) .
\end{aligned}
$$

From the above expressions one can see that $P(\mu)$ is continuous and convex as $e(\rho)$, while $\mu(\rho)$ and $P(\rho)$ have a jump in $\rho_{K}$ (see Fig. 6). Therefore the glass transition, which is a second order transition in the classical case, looks like a first order transition in the zero-temperature quantum problem. This is similar to previous results on quantum meanfield glass models with quenched disorder (see Refs. 66 and 67). On the other hand the properties of the first order transition are quite different. In the case of mean-field quantum glass models the glass and liquid are really different phases and the glass phase does not appear (via density fluctuations that are frozen on time scales diverging at the transition) in a continuous way from the liquid, contrary to what happens for the superglass.

The sound velocity is now determined by 

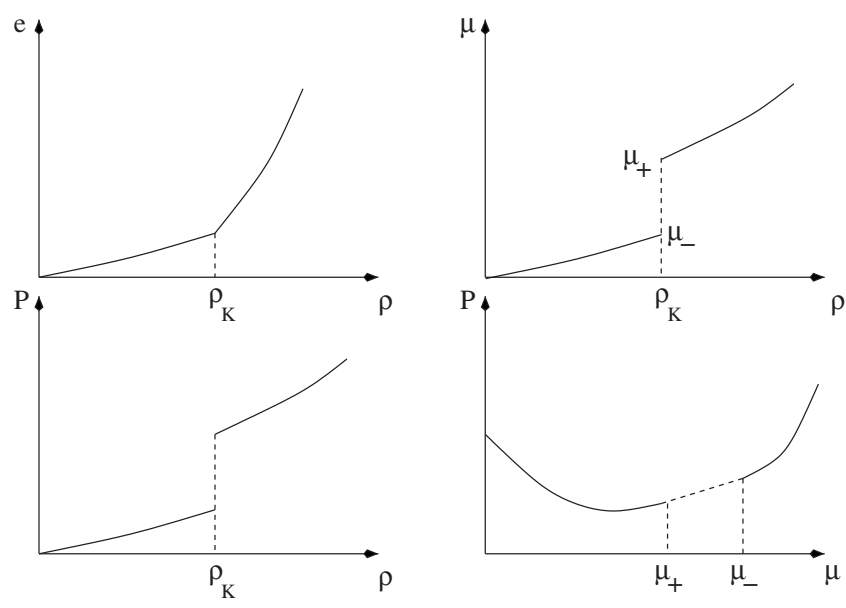

FIG. 6. Schematic behavior of $e(\rho), P(\mu), \mu(\rho)$, and $P(\rho)$ across the glass transition. Note that there are values of $P$ and $\mu$ that correspond to the same density $\rho_{K}$.

$$
c^{2}=\frac{1}{\rho \chi_{T}}=\frac{d P}{d \rho}=\frac{d}{d \rho}\left[\rho^{2} \frac{d e}{d \rho}\right] \neq 0 .
$$

General arguments ${ }^{68}$ show that if $c \neq 0$, then $S(k)$ $\leq k /(2 m c)$; assuming equality the Feynman formula gives $\epsilon(k)=c k$, consistently with the existence of sound waves.

Therefore the system in this case has a finite critical velocity and is superfluid. Note that in the glass the first peak of the structure factor, which determines $\min _{k}[\epsilon(k) / k]$, is close to the one of the liquid, suggesting that the critical velocity should stay close to the one of the liquid. Still it is known (e.g., in helium) that the precise determination of the critical velocity is complicated and depends on the geometry; therefore estimating precisely its value in the superglass phase is beyond the scope of this paper.

\section{CONCLUSION}

In this paper we presented and analyzed a concrete model of interacting bosons that displays unambiguously, in addition to superfluid and supercrystal phases, metastable superfluid and superglass phases. This shows concretely that a system can be at the same time glassy, i.e., displaying very slow dynamics for the structural degrees of freedom, and supersolid, i.e., showing a superfluid component. Note that there is no paradox, exactly as there is none for the supercrystal. Indeed, Leggett ${ }^{3}$ in his original paper "Can a solid be superfluid?" explicitly mentioned the possibility of superamorphous solids.

The exact ground state of the model we considered is known by construction: it is a Jastrow wave function ${ }^{43}$ with a hard sphere potential form. We constructed the quantum Hamiltonian for which this wave function is exact via a mapping to Brownian motion of classical hard spheres, following the connection between the Fokker-Planck operator for the classical stochastic dynamics and the Schrödinger operator describing the associated quantum system. This mapping allows us to understand the physics of the superglass phase by using results from the well-studied problem of densely packed hard spheres.
Our findings, which were summarized in Sec. II, are most simply conveyed through Fig. 2. By changing the density of particles, one goes from a superfluid phase to a supercrystal via a first order phase transition. If the density is increased fast or in case of (binary) mixtures, one reaches a metastable superfluid state, and if the density is further increased, one reaches the superglass phase.

The analysis we carried out for the hard sphere Jastrow wave function ${ }^{43}$ can be extended for other systems for which the Jastrow wave function ${ }^{43}$ corresponds to a classical potential leading to glassy dynamics. Classically, mixtures may become glasses at certain compositions, for example, $80 \%$ / $20 \%$ mixtures of two species of particles interacting via Lennard-Jones potentials. ${ }^{70}$ The problem of binary mixtures is interesting in that one could potentially realize the system in cold atomic gases, where the relative fractions of two species could be controlled. We have worked out the correspondence between the classical and quantum problems for binary mixtures in Appendix. Following the same reasoning as in our work on the hard sphere wave functions, the binary mixtures can display superglass behavior as long as the mapped classical problem does so.

The usefulness of the classical to quantum mapping is that it allows us to make precise and concrete statements about the nature of the ground state of the devised Hamiltonian. By construction, the ground-state energy is exactly zero, and thus one must add a small perturbation so as to obtain a nonzero speed of sound, as we have done in Sec. VI. We have added a two-body potential as perturbation because we could make use of the knowledge of the density-density correlation $g(r)$ in obtaining the effect of the additional interaction on the ground-state energy, pressure, compressibility, and sound velocity of the system. It is an interesting possibility that if, as opposed to the two-body perturbation, one adds a three-body potential, one could compensate the threebody term that arises from the classical to quantum mapping. In other words, we speculate that by removing (or reducing) the three-body term by a compensating perturbation, one could achieve a quantum Hamiltonian with a glassy ground state, only two-body terms, and a finite sound velocity. This could perhaps be tested numerically by considering the system with the potential in Eq. (12) alone. Moreover, if the short-ranged "sticky" part of Eq. (12) potential is removed, this may not substantially change the results. At least, we expect the system to be glassy as the original one (the question of superfluidity is instead a more tricky one and needs further investigations). The intuitive reason is as follows: for dense packings and a hard sphere potential, if one turns down the kinetic energy (say by "increasing" the mass of the particles), thus making the system more classical, one should recover the physics of the classical hard sphere system. Because the packings are dense, turning up the kinetic energy (say by "decreasing" the mass of the particles) should not favor very much transitions between a glassy state $\alpha$ to another $\beta$. Because of the hard sphere potential (basically infinite on the scales of the kinetic energy), we do not expect, at a dense packing, that quantum fluctuations will reduce considerably (at least for not too small $\mathrm{m}$ ) the time scales to escape from classical glassy configurations.

Concerning the superglass transition, we found it to be first order but quite unusual. It is different from the quantum 
first order transitions found for mean-field glassy models in Refs. 66 and 67. It would be interesting to develop and study mean-field models (or mean-field approximations) able to reproduce our results and the superglass phase transitions. One of the main motivations is that they could allow one to analyze the finite temperature regime which is clearly out of reach of the approach developed in this work. In particular, it would be very interesting to apply quantum mode-coupling theory $^{71}$ (QMCT) to the model we focus on. It would be very interesting to know whether QMCT predicts quantum correlation functions that are connected to the one predicted by classical MCT via the classical-quantum-mapping we employed. If yes, this would mean that QMCT is able to capture this unusual first order phase transition toward the superglass phase and it would allow one to study its finite temperature extension.

Finally, let us discuss preliminary results for $\mathrm{He}^{4}$ which is one of the original motivations of our work. Jastrow wave functions $^{43}$ have been used extensively to describe the ground state of $\mathrm{He}^{4}$ in both fluid ${ }^{44,45}$ and solid ${ }^{46}$ phases. Therefore it is tempting to try to study the superglass phase of $\mathrm{He}^{4}$ by using the Jastrow wave function ${ }^{43}$ as a variational wave function. For $\mathrm{He}^{4}$ it was found that the liquid phase is well described by a potential $V(r) \sim r^{-5}$. We then computed the glass transition density for this potential following Ref. 69. We found that the superglass transition takes place at a density $\rho_{K} \sim 0.45 \AA^{-3}$ and a very small jump in the first derivative of $e(\rho)$ at $\rho_{K}$, the relative variation in $e^{\prime}(\rho)$ being of the order of $10^{-3}$. Unfortunately, the value of $\rho_{K}$ is ten times higher than the density at which the superglass has been observed in Ref. 20. However, it is well known that the Jastrow wave function ${ }^{43}$ overestimates the liquid-crystal transition for $\mathrm{He}^{4}$ and, more generally, provides a poor description of the solid phases. A more refined investigation should involve shadow-like wave functions, ${ }^{72}$ and we plan to report on such a study in a future publication where more details on the Jastrow wave function ${ }^{43}$ will also be given.

\section{ACKNOWLEDGMENTS}

We thank S. Balibar, S. Baroni, M. Boninsegni, J.-P. Bouchaud, G. Carleo, C. Castelnovo, A. Lefèvre, S. Moroni, O. Parcollet, L. Reatto, D. R. Reichman, M. Tarzia, and X. Wayntal for discussions. We also thank R. Di Leonardo for bringing Ref. 63 to our attention. G.B. work is supported by ANR grant DYNHET. F.Z. acknowledges financial support from the ESF Research Networking Programme INSTANS.

\section{APPENDIX: QUANTUM-CLASSICAL MAPPING FOR A BINARY MIXTURE OF BOSONS}

Here we work out the details of the connection between the quantum and classical fluids for a mixture of two types of particles, $A$ and $B$. We start again with the Jastrow-type wave function ${ }^{43}$ written in terms of the interaction among $N=N_{A}$ $+N_{B}$ particles, through a potential

$$
\begin{aligned}
U(\{\mathbf{x}\} ;\{\mathbf{X}\}) \equiv & U\left(\mathbf{x}_{1}, \ldots, \mathbf{x}_{N_{A}} ; \mathbf{X}_{1}, \ldots, \mathbf{X}_{N_{B}}\right)=\sum_{i>j} V_{A A}\left(\left|\mathbf{x}_{i}-\mathbf{x}_{j}\right|\right) \\
& +\sum_{I>J} V_{B B}\left(\left|\mathbf{X}_{I}-\mathbf{X}_{J}\right|\right)+\sum_{i, I} V_{A B}\left(\left|\mathbf{x}_{i}-\mathbf{X}_{J}\right|\right), \quad(\mathrm{A} 1)
\end{aligned}
$$

where $i, j=1, \ldots, N_{A}$ and $I, J=1, \ldots, N_{B}$. The dynamics of a classical mixture of particles interacting through this potential is clearly a particular case of the general Langevin equation specified by Eqs. (1) and (2), where we set $\gamma_{i}=\gamma_{A}$ and $\gamma_{I}=\gamma_{B}$, and the form of the Fokker-Planck operator follows straightforwardly from Eq. (4).

The quantum Hamiltonian is, from Eq. (7),

$$
H=\sum_{i} \frac{p_{i}^{2}}{2 m_{A}}+\sum_{I} \frac{P_{I}^{2}}{2 m_{B}}+\mathcal{V}(\{\mathbf{x}\} ;\{\mathbf{X}\}),
$$

where $m_{A, B}=\hbar^{2} \gamma_{A, B} /(2 T)$ and, setting $T=1$ and $\hbar=1$,

$$
\begin{aligned}
\mathcal{V}(\{\mathbf{x}\} ;\{\mathbf{X}\}) \equiv & \mathcal{V}\left(\mathbf{x}_{1}, \ldots, \mathbf{x}_{N_{A}} ; \mathbf{X}_{1}, \ldots, \mathbf{X}_{N_{B}}\right) \\
= & \frac{1}{4 m_{A}} \sum_{i}\left\{\frac{1}{2}\left(\nabla_{i} U\right)^{2}-\nabla_{i}^{2} U\right\} \\
& +\frac{1}{4 m_{B}} \sum_{I}\left\{\frac{1}{2}\left(\nabla_{I} U\right)^{2}-\nabla_{I}^{2} U\right\} .
\end{aligned}
$$

The ground state of this Hamiltonian is

$$
\Psi_{G}(\{\mathbf{x}\} ;\{\mathbf{X}\})=\frac{1}{\sqrt{Z_{N_{A}, N_{B}}}} \exp \left[-\frac{1}{4} U(\{\mathbf{x}\} ;\{\mathbf{X}\})\right] .
$$

The quantum potential $\mathcal{V}(\{\mathbf{x}\} ;\{\mathbf{X}\})$ will again have contributions in the form of two-body and three-body terms,

$$
\begin{aligned}
\mathcal{V}= & \sum_{i>j} v_{i j}^{A A \text { pair }}+\sum_{I>J} v_{I J}^{B B} \text { pair }+\sum_{i, I} v_{i I}^{A B} \text { pair } \\
& + \text { three-body terms. }
\end{aligned}
$$

The pair potential is a simple generalization of Eq. (11),

$$
v_{i j}^{A A \text { pair }}=\frac{1}{2 m_{A}}\left\{-\frac{d-1}{r_{i j}} V_{A A}^{\prime}\left(r_{i j}\right)-V_{A A}^{\prime \prime}\left(r_{i j}\right)+\frac{1}{2}\left[V_{A A}^{\prime}\left(r_{i j}\right)\right]^{2}\right\} \text {, }
$$

$$
\begin{aligned}
& v_{I J}^{B B} \text { pair }=\frac{1}{2 m_{B}}\left\{-\frac{d-1}{r_{I J}} V_{B B}^{\prime}\left(r_{I J}\right)-V_{B B}^{\prime \prime}\left(r_{I J}\right)+\frac{1}{2}\left[V_{B B}^{\prime}\left(r_{I J}\right)\right]^{2}\right\}, \\
& v_{i I}^{A B} \text { pair }=\frac{m_{A}+m_{B}}{4 m_{A} m_{B}}\left\{-\frac{d-1}{r_{i I}} V_{A B}^{\prime}\left(r_{i I}\right)-V_{A B}^{\prime \prime}\left(r_{i I}\right)\right. \\
& \left.+\frac{1}{2}\left[V_{A B}^{\prime}\left(r_{i I}\right)\right]^{2}\right\} \text {, }
\end{aligned}
$$

and similarly for the three-body terms.

\section{Quantum model associated with Lennard-Jones binary mixtures}

Let us construct the quantum model associated to a Lennard-Jones binary mixture, for which the underlying classical system can be glassy, for instance, certain $80 \% / 20 \%$ mixtures of $A / B$ particles. ${ }^{70}$ Let us consider classical potentials of the form $V_{P_{1} P_{2}}(r)=-\varepsilon_{P_{1} P_{2}}\left[\left(r / \sigma_{P_{1} P_{2}}\right)^{-\alpha}-\left(r / \sigma_{P_{1} P_{2}}\right)^{-\beta}\right]$, where $P_{1}, P_{2}=A$ or $B$, with three energy scales, $\varepsilon_{A A}, \varepsilon_{B B}$, and $\varepsilon_{A B}$, and three characteristic lengths, $\sigma_{A A}, \sigma_{B B}$, and $\sigma_{A B}$, in 
the problem. The exponents $\beta>\alpha>0$ make the classical potential attractive at long distances and repulsive at short ones. The corresponding quantum pair potential is

$$
\begin{aligned}
v^{P_{1} P_{2} \operatorname{pair}}(r)= & \frac{m_{P_{1}}+m_{P_{2}}}{4 m_{P_{1}} m_{P_{2}}} \frac{\varepsilon_{P_{1} P_{2}}}{\sigma_{P_{1} P_{2}}^{2}}\left\{\left[\alpha(\alpha+2-d)\left(r / \sigma_{P_{1} P_{2}}\right)^{-(\alpha+2)}\right]\right. \\
& -\left[\beta(\beta+2-d)\left(r / \sigma_{P_{1} P_{2}}\right)^{-(\beta+2)}\right] \\
& +\varepsilon_{P_{1} P_{2}}\left[\frac{\alpha^{2}}{2}\left(r / \sigma_{P_{1} P_{2}}\right)^{-(2 \alpha+2)}\right. \\
& \left.\left.-\alpha \beta\left(r / \sigma_{P_{1} P_{2}}\right)^{-(\alpha+\beta+2)}+\frac{\beta^{2}}{2}\left(r / \sigma_{P_{1} P_{2}}\right)^{-(2 \beta+2)}\right]\right\} .
\end{aligned}
$$

Notice that the pair potential is repulsive for both small and large distances: $v^{P_{1} P_{2}}$ pair $(r) \sim\left(r / \sigma_{P_{1} P_{2}}\right)^{-(\alpha+2)}$ for $r / \sigma_{P_{1} P_{2}} \gg 1$ and $v^{P_{1} P_{2}} \operatorname{pair}(r) \sim\left(r / \sigma_{P_{1} P_{2}}\right)^{-(2 \beta+2)}$ for $r / \sigma_{P_{1} P_{2}} \ll 1$. At intermediate distances, $r / \sigma_{P_{1} P_{2}} \sim 1$, the potential can become attractive, as illustrated in Fig. 7 starting from an $\alpha=6$ and $\beta$ $=12$ classical Lennard-Jones potential.

\section{Off-diagonal long-range order in binary mixtures}

We can extend the Penrose-Onsager ${ }^{48}$ definition of offdiagonal long-range order to a mixture of two distinct types of bosonic atoms. Let

$$
\begin{aligned}
\mathcal{R}_{A}\left(\mathbf{x}-\mathbf{x}^{\prime}\right) & \\
= & V \int \prod_{i=2}^{N_{A}} d \mathbf{x}_{i} \prod_{I=1}^{N_{B}} d \mathbf{X}_{I} \Psi_{G}\left(\mathbf{x}, \mathbf{x}_{2}, \ldots, \mathbf{x}_{N_{A}} ; \mathbf{X}_{1}, \ldots, \mathbf{X}_{N_{B}}\right) \\
& \times \Psi_{G}\left(\mathbf{x}^{\prime}, \mathbf{x}_{2}, \ldots, \mathbf{x}_{N_{A}} ; \mathbf{X}_{1}, \ldots, \mathbf{X}_{N_{B}}\right)
\end{aligned}
$$

and

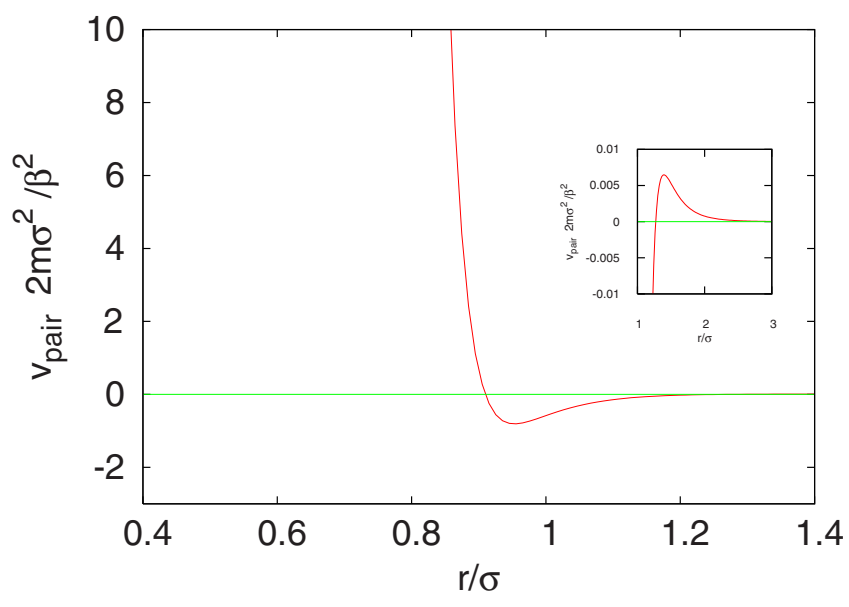

FIG. 7. (Color online) Form of the pair potential $v^{\text {pair }}(r)$ in the mapped quantum problem that derives from the classical $\alpha=6$ and $\beta=12$ Lennard-Jones potential. ( $\varepsilon=1$ is set for simplicity.) The potential is repulsive at large and small distances, but to see the repulsion for large $r$ one needs to zoom closer, as shown in the inset.

$$
\begin{aligned}
\mathcal{R}_{B}\left(\mathbf{X}-\mathbf{X}^{\prime}\right) & \\
= & V \int \prod_{i=1}^{N_{A}} d \mathbf{x}_{i} \prod_{I=2}^{N_{B}} d \mathbf{X}_{I} \Psi_{G}\left(\mathbf{x}_{1}, \ldots, \mathbf{x}_{N_{A}} ; \mathbf{X}, \mathbf{X}_{2}, \ldots, \mathbf{X}_{N_{B}}\right) \\
& \times \Psi_{G}\left(\mathbf{x}_{1}, \ldots, \mathbf{x}_{N_{A}} ; \mathbf{X}^{\prime}, \mathbf{X}_{2}, \ldots, \mathbf{X}_{N_{B}}\right)
\end{aligned}
$$

We can write these expressions in terms of the classical liquid correlations,

$$
\begin{aligned}
& \mathcal{R}_{A}\left(\mathbf{x}-\mathbf{x}^{\prime}\right)=V \frac{1}{Z_{N_{A}, N_{B}}} \int \prod_{i=2}^{N_{A}} d \mathbf{x}_{i} \prod_{I=1}^{N_{B}} d \mathbf{X}_{I} \exp \left[-U\left(\mathbf{x}_{2}, \ldots, \mathbf{x}_{N_{A}} ; \mathbf{X}_{1}, \ldots, \mathbf{X}_{N_{B}}\right)\right] \\
& \times \exp \left\{-\frac{1}{2}\left[\sum_{j=2}^{N_{A}} V_{A A}\left(\left|\mathbf{x}-\mathbf{x}_{j}\right|\right)+\sum_{J=1}^{N_{B}} V_{A B}\left(\left|\mathbf{x}-\mathbf{X}_{J}\right|\right)\right]\right\} \exp \left\{-\frac{1}{2}\left[\sum_{j=2}^{N_{A}} V_{A A}\left(\left|\mathbf{x}^{\prime}-\mathbf{x}_{j}\right|\right)+\sum_{J=1}^{N_{B}} V_{A B}\left(\left|\mathbf{x}^{\prime}-\mathbf{X}_{J}\right|\right)\right]\right\} \\
& =V \frac{Z_{N_{A}-1, N_{B}}}{Z_{N_{A}, N_{B}}} \int \prod_{i=2}^{N_{A}} d \mathbf{x}_{i} \prod_{I=1}^{N_{B}} d \mathbf{X}_{I} P_{G}\left(\mathbf{x}_{2}, \ldots, \mathbf{x}_{N_{A}} ; \mathbf{X}_{1}, \ldots, \mathbf{X}_{N_{B}}\right) \\
& \times \exp \left\{-\frac{1}{2}\left[\sum_{j=2}^{N_{A}} V_{A A}\left(\left|\mathbf{x}-\mathbf{x}_{j}\right|\right)+\sum_{J=1}^{N_{B}} V_{A B}\left(\left|\mathbf{x}-\mathbf{X}_{J}\right|\right)\right]\right\} \exp \left\{-\frac{1}{2}\left[\sum_{j=2}^{N_{A}} V_{A A}\left(\left|\mathbf{x}^{\prime}-\mathbf{x}_{j}\right|\right)+\sum_{J=1}^{N_{B}} V_{A B}\left(\left|\mathbf{x}^{\prime}-\mathbf{X}_{J}\right|\right)\right]\right\} \\
& =V \frac{Z_{N_{A}-1, N_{B}}}{Z_{N_{A}, N_{B}}}\left\langle e^{-(1 / 2) \Phi_{A}(\mathbf{x})} e^{-(1 / 2) \Phi_{A}\left(\mathbf{x}^{\prime}\right)}\right\rangle
\end{aligned}
$$


where we defined the potential caused by the $N_{A}-1$ type $A$ particles and the $N_{B}$ type $B$ ones as

$$
\Phi_{A}(\mathbf{x})=\int d \mathbf{r}\left[V_{A A}(|\mathbf{x}-\mathbf{r}|) \rho_{A}(\mathbf{r})+V_{A B}(|\mathbf{x}-\mathbf{r}|) \rho_{B}(\mathbf{r})\right],
$$

with the densities given by $\rho_{A}(\mathbf{r})=\sum_{j=2}^{N_{A}} \delta\left(\mathbf{r}-\mathbf{x}_{j}\right)$ and $\rho_{B}(\mathbf{r})$ $=\sum_{J=1}^{N_{B}} \delta\left(\mathbf{r}-\mathbf{x}_{j}\right)$.

Similarly, one obtains

$$
\mathcal{R}_{B}\left(\mathbf{X}-\mathbf{X}^{\prime}\right)=V \frac{Z_{N_{A}, N_{B}-1}}{Z_{N_{A}, N_{B}}}\left\langle e^{-(1 / 2) \Phi_{B}(\mathbf{X})} e^{-(1 / 2) \Phi_{B}\left(\mathbf{X}^{\prime}\right)}\right\rangle,
$$

where the potential caused by the $N_{A}$ type $A$ particles and the $N_{B}-1$ type $B$ is given by

$$
\Phi_{B}(\mathbf{X})=\int d \mathbf{r}\left[V_{A B}(|\mathbf{X}-\mathbf{r}|) \rho_{A}(\mathbf{r})+V_{B B}(|\mathbf{X}-\mathbf{r}|) \rho_{B}(\mathbf{r})\right]
$$

One can define the condensate fraction of type $A$ bosons as $n_{A}=\mathcal{R}_{A}(\infty) \equiv \lim _{\left|\mathbf{x}-\mathbf{x}^{\prime}\right| \rightarrow \infty} \mathcal{R}_{A}\left(\mathbf{x}-\mathbf{x}^{\prime}\right)$, and so

$$
n_{A}=V \frac{Z_{N_{A}-1, N_{B}}}{Z_{N_{A}, N_{B}}}\left\langle e^{-(1 / 2) \Phi_{A}(\mathbf{0})}\right\rangle^{2},
$$

where we assumed that the two-point correlation function factorizes,

$$
\begin{aligned}
\left\langle e^{-(1 / 2) \Phi_{A}(\mathbf{x})} e^{-(1 / 2) \Phi_{A}\left(\mathbf{x}^{\prime}\right)}\right\rangle & \rightarrow\left\langle e^{-(1 / 2) \Phi_{A}(\mathbf{x})}\right\rangle\left\langle e^{-(1 / 2) \Phi_{A}\left(\mathbf{x}^{\prime}\right)}\right\rangle \\
& =\left\langle e^{-(1 / 2) \Phi_{A}(\mathbf{0})}\right\rangle^{2},
\end{aligned}
$$

and also that the one-point function is translational invariant. Notice that the factorization assumption should be fine for short-ranged potentials or potentials that decay sufficiently fast, but there may be certain (possibly pathological) potentials for which it may fail.

One can check that this definition of the condensate fraction of $A$ bosons is such that $n_{A} \leq 1$ as follows. First we use Hölder's inequality to obtain

$$
\left\langle e^{-(1 / 2) \Phi_{A}(\mathbf{x})} e^{-(1 / 2) \Phi_{A}\left(\mathbf{x}^{\prime}\right)}\right\rangle \leq \sqrt{\left\langle e^{-\Phi_{A}(\mathbf{x})}\right\rangle\left\langle e^{-\Phi_{A}\left(\mathbf{x}^{\prime}\right)}\right\rangle}=\left\langle e^{-\Phi_{A}(\mathbf{0})}\right\rangle,
$$

from which we conclude that $\mathcal{R}_{A}\left(\mathbf{x}-\mathbf{x}^{\prime}\right) \leq \mathcal{R}_{A}(\mathbf{0})=1$, the last equality following trivially from the normalized wave functions (plus translational invariance). Thus, $n_{A} \leq 1$ follows from the definitions above. Similar results apply to $n_{B}$. Notice that $\mathcal{R}_{A}(\mathbf{0})=1$ is a simple way to obtain $\left\langle e^{-\Phi_{A}(\mathbf{0})}\right\rangle$ $=\frac{1}{V} \frac{Z_{N_{A}, N_{B}}}{Z_{N_{A}-1, N_{B}}}$; we will use this equality below.

\section{a. Fixing the chemical potential}

If we work at fixed chemical potential, we can relate the condensate fraction of bosons $A$ and $B$ to the fugacities $z_{A, B}$ and the densities $\rho_{A, B}$ as follows. The grand canonical partition function for the binary mixture is given by

$$
\mathcal{Z}=\sum_{N_{A}, N_{B}} \frac{z_{A}^{N_{A}}}{N_{A} !} \frac{z_{B}^{N_{B}}}{N_{B} !} Z_{N_{A}, N_{B}}
$$

At the saddle point, with particle numbers $N_{A}^{*}$ and $N_{B}^{*}$, one has the relations

$$
\frac{N_{A}^{*}}{z_{A}} Z_{N_{A}^{*}-1, N_{B}^{*}}=Z_{N_{A}^{*}, N_{B}^{*}}=\frac{N_{B}^{*}}{z_{B}} Z_{N_{A}^{*}, N_{B}^{*}-1} .
$$

Substitution of these relations in the equations for $n_{A, B}$ allows us to write

$$
n_{A}=\frac{z_{A}}{\rho_{A}}\left\langle e^{-(1 / 2) \Phi_{A}(\mathbf{0})}\right\rangle^{2}
$$

and

$$
n_{B}=\frac{z_{B}}{\rho_{B}}\left\langle e^{-(1 / 2) \Phi_{B}(\mathbf{0})}\right\rangle^{2} .
$$

Notice that Eq. (A19) also allows us to write

$$
\left\langle e^{-\Phi_{A}(\mathbf{0})}\right\rangle=\frac{1}{V} \frac{Z_{N_{A}, N_{B}}}{Z_{N_{A}-1, N_{B}}}=\frac{\rho_{A}}{z_{A}}, \quad\left\langle e^{-\Phi_{B}(\mathbf{0})}\right\rangle=\frac{1}{V} \frac{Z_{N_{A}, N_{B}}}{Z_{N_{A}, N_{B}-1}}=\frac{\rho_{B}}{z_{B}}
$$

at equilibrium.

These relations allow us further to put lower bounds on the condensate fraction. To do so, consider without loss of generality potentials such that $\Phi_{A, B}(\mathbf{0}) \geq 0$; one can always do so by shifting the energies by a constant value such that the potentials $V_{A A}, V_{B B}$, and $V_{A B}$ are non-negative (and using that the densities $\left.\rho_{A, B} \geq 0\right)$. In this case, we can write

$$
\left\langle e^{-(1 / 2) \Phi_{A, B}(\mathbf{0})}\right\rangle \geq\left\langle e^{-\Phi_{A, B}(\mathbf{0})}\right\rangle=\frac{\rho_{A, B}}{z_{A, B}}
$$

and thus

$$
n_{A, B} \geq \frac{\rho_{A, B}}{z_{A, B}} .
$$

For a hard sphere potentials, the equality $\left\langle e^{-(1 / 2) \Phi_{A, B}^{\text {hard }}(\mathbf{0})}\right\rangle$ $=\left\langle e^{-\Phi_{A, B}^{\text {hard }}(\mathbf{0})}\right\rangle$ is satisfied. Thus, hard spheres have the lowest possible condensate fractions respecting the bound $[\mathrm{Eq}$. (A23)]. 
${ }^{1}$ H. A. Barnes, J. Non-Newtonian Fluid Mech. 81, 133 (1999).

${ }^{2}$ A. F. Andreev and I. M. Lifshitz, Sov. Phys. JETP 29, 1107 (1969).

${ }^{3}$ A. J. Leggett, Phys. Rev. Lett. 25, 1543 (1970).

${ }^{4}$ G. V. Chester, Phys. Rev. A 2, 256 (1970).

${ }^{5}$ E. Kim and M. H. W. Chan, Nature (London) 427, 225 (2004); Science 305, 1941 (2004).

${ }^{6}$ A. C. Clark, J. T. West, and M. H. W. Chan, Phys. Rev. Lett. 99, 135302 (2007).

${ }^{7}$ M. W. Ray and R. B. Hallock, Phys. Rev. Lett. 100, 235301 (2008).

${ }^{8}$ For reviews, see: D. Ceperley, Nat. Phys. 2, 659 (2006); N. V. Prokof'ev, Adv. Phys. 56, 381 (2007); P. Phillips and A. Balatsky, Science 316, 1435 (2007); S. Balibar and F. Caupin, J. Phys.: Condens. Matter 20, 173201 (2008).

${ }^{9}$ D. M. Ceperley and B. Bernu, Phys. Rev. Lett. 93, 155303 (2004); B. K. Clark and D. M. Ceperley, ibid. 96, 105302 (2006).

${ }^{10}$ M. Boninsegni, A. B. Kuklov, L. Pollet, N. V. Prokof'ev, B. V. Svistunov, and M. Troyer, Phys. Rev. Lett. 97, 080401 (2006).

${ }^{11}$ E. Vitali, M. Rossi, F. Tramonto, D. E. Galli, and L. Reatto, Phys. Rev. B 77, 180505(R) (2008).

${ }^{12}$ C. Josserand, Y. Pomeau, and S. Rica, Phys. Rev. Lett. 98, 195301 (2007).

${ }^{13}$ Ann Sophie C. Rittner and J. D. Reppy, Phys. Rev. Lett. 97, 165301 (2006).

${ }^{14}$ Ann Sophie C. Rittner and J. D. Reppy, Phys. Rev. Lett. 98, 175302 (2007).

${ }^{15}$ M. Boninsegni, A. B. Kuklov, L. Pollet, N. V. Prokofev, B. V. Svistunov, and M. Troyer, Phys. Rev. Lett. 99, 035301 (2007).

${ }^{16}$ J. Toner, Phys. Rev. Lett. 100, 035302 (2008).

${ }^{17}$ P. G. de Gennes, C. R. Phys. 7, 561 (2006).

${ }^{18}$ J.-P. Bouchaud and G. Biroli, arXiv:0710.3087, C. R. Phys. (to be published).

${ }^{19}$ S. Sasaki, R. Ishiguro, F. Caupin, H. J. Maris, and S. Balibar, Science 313, 1098 (2006).

${ }^{20}$ M. Boninsegni, N. Prokof'ev, and B. Svistunov, Phys. Rev. Lett. 96, 105301 (2006).

${ }^{21}$ A. V. Balatsky, M. J. Graf, Z. Nussinov, and S. A. Trugman, Phys. Rev. B 75, 094201 (2007).

${ }^{22}$ G. Parisi, Statistical Field Theory (Addison-Wesley, New York, 1988).

${ }^{23}$ J. Zinn-Justin, Quantum Field Theory and Critical Phenomena (Oxford University Press, New York, 2002).

${ }^{24}$ D. S. Rokhsar and S. A. Kivelson, Phys. Rev. Lett. 61, 2376 (1988).

${ }^{25}$ C. L. Henley, J. Phys.: Condens. Matter 16, S891 (2004).

${ }^{26}$ C. Castelnovo, C. Chamon, C. Mudry, and P. Pujol, Ann. Phys. (N.Y.) 318, 316 (2005).

${ }^{27}$ W. G. Hoover and F. H. Ree, J. Chem. Phys. 49, 3609 (1968).

${ }^{28}$ N. Prokof'ev and B. Svistunov, Phys. Rev. Lett. 94, 155302 (2005).

${ }^{29}$ T. R. Kirkpatrick and P. G. Wolynes, Phys. Rev. A 35, 3072 (1987).

${ }^{30}$ W. van Megen and S. M. Underwood, Phys. Rev. Lett. 70, 2766 (1993).

${ }^{31}$ R. J. Speedy, Mol. Phys. 95, 169 (1998).

${ }^{32}$ M. Cardenas, S. Franz, and G. Parisi, J. Chem. Phys. 110, 1726 (1999).

${ }^{33}$ G. Parisi and F. Zamponi, arXiv:0802.2180 (unpublished).
${ }^{34}$ G. Parisi and F. Zamponi, J. Chem. Phys. 123, 144501 (2005).

${ }^{35}$ L. Santen and W. Krauth, arXiv:cond-mat/0107459 (unpublished).

${ }^{36}$ P. Chaudhuri, S. Karmakar, C. Dasgupta, H. R. Krishnamurthy, and A. K. Sood, Phys. Rev. Lett. 95, 248301 (2005).

${ }^{37}$ D. M. Kagan, M. Feigel'man, and L. B. Ioffe, Zh. Eksp. Teor. Fiz. 116, 1450 (1999) [JETP 89, 781 (1999)].

${ }^{38}$ H. Westfahl, Jr., J. Schmalian, and P. G. Wolynes, Phys. Rev. B 68, 134203 (2003).

${ }^{39}$ C. Chamon, Phys. Rev. Lett. 94, 040402 (2005).

${ }^{40}$ C. Castelnovo, C. Chamon, C. Mudry, and P. Pujol, Phys. Rev. B 72, 104405 (2005).

${ }^{41}$ M. Tarzia and G. Biroli, Europhys. Lett. 82, 67008 (2008).

${ }^{42}$ S. Baroni and S. Moroni, Phys. Rev. Lett. 82, 4745 (1999).

${ }^{43}$ R. Jastrow, Phys. Rev. 98, 1479 (1955).

${ }^{44}$ W. L. McMillan, Phys. Rev. 138, A442 (1965).

${ }^{45}$ W. P. Francis, G. V. Chester, and L. Reatto, Phys. Rev. A 1, 86 (1970).

${ }^{46}$ J.-P. Hansen and D. Levesque, Phys. Rev. 165, 293 (1968).

${ }^{47}$ See, e.g., Liquid, Freezing and Glass Transition, Les Houches Session LI, 1989, edited by J.-P. Hansen, D. Levesque, and J. Zinn-Justin (Elsevier, Amsterdam, 1990).

${ }^{48}$ O. Penrose and L. Onsager, Phys. Rev. 104, 576 (1956).

${ }^{49}$ A. J. Leggett, Quantum Liquids (Oxford University Press, New York, 2006).

${ }^{50}$ J.-P. Hansen and I. R. MacDonald, Theory of Simple Liquids (Academic, London, 1986).

${ }^{51}$ D. A. Young and B. J. Adler, J. Chem. Phys. 60, 1254 (1974).

${ }^{52}$ A. R. Denton, N. W. Ashcroft, and W. A. Curtin, Phys. Rev. E 51, 65 (1995).

${ }^{53}$ R. J. Speedy, J. Phys.: Condens. Matter 10, 4387 (1998).

${ }^{54}$ M. Skoge, A. Donev, F. H. Stillinger, and S. Torquato, Phys. Rev. E 74, 041127 (2006).

${ }^{55}$ P. N. Pusey and W. van Megen, Phys. Rev. Lett. 59, 2083 (1987).

${ }^{56}$ E. R. Weeks, J. C. Crocker, A. C. Levitt, A. Schofield, and D. A. Weitz, Science 287, 627 (2000)

${ }^{57}$ W. Götze, J. Phys.: Condens. Matter 11, A1 (1999).

${ }^{58}$ M. Mézard and G. Parisi, Phys. Rev. Lett. 82, 747 (1999).

${ }^{59}$ C. M. Newman and D. S. Stein, J. Stat. Phys. 106, 213 (2002).

${ }^{60}$ J. P. Bouchaud and G. Biroli, J. Chem. Phys. 121, 7347 (2004).

${ }^{61}$ A. Montanari and G. Semerjian, J. Stat. Phys. 125, 23 (2006).

${ }^{62}$ D. Forster, Hydrodynamics Fluctuations, Broken Symmetry, and Correlation Functions (Perseus Books, 1990).

${ }^{63}$ A. Bello, E. Laredo, and M. Grimau, Phys. Rev. B 60, 12764 (1999).

${ }^{64}$ L. D. Landau, E. M. Lifshitz, and L. P. Pitaevski, Course of Theoretical Physics, Statistical Physics Vol. 9 (Pergamon, Oxford, 1980), Pt. 2.

${ }^{65}$ A. A. Abrikosov, L. P. Gorkov, and I. E. Dzyaloshinski, Methods of Quantum Field Theory in Statistical Physics (Dover, New York, 1975).

${ }^{66}$ L. F. Cugliandolo, D. R. Grempel, and C. A. da Silva Santos, Phys. Rev. B 64, 014403 (2001).

${ }^{67}$ G. Biroli and L. F. Cugliandolo, Phys. Rev. B 64, 014206 (2001).

${ }^{68}$ See Sec. 13.7 of K. Huang, Statistical Mechanics (Wiley, New York, 1987).

${ }^{69}$ M. Cardenas and M. P. Tosi, Phys. Lett. A 336, 423 (2005).

${ }^{70}$ W. Kob and H. C. Andersen, Phys. Rev. E 51, 4626 (1995); 52, 
4134 (1995)

${ }^{71}$ E. Rabani and D. R. Reichman, Annu. Rev. Phys. Chem. 56, 157 (2005).

${ }^{72}$ T. MacFarland, S. A. Vitiello, L. Reatto, G. V. Chester, and M. H. Kalos, Phys. Rev. B 50, 13577 (1994).

${ }^{73}$ It is worth to stress that in general Eq. (30) is not a decomposition in pure states of a Gibbs measure like Eq. (27). The crucial difference is that in Eq. (27) the $P_{\alpha}$ are normalized to 1 and the weights $w_{\alpha}$ add up to one, while this is not the case in Eq. (30).

${ }^{74}$ There is only one step that is not detailed in Refs. 33 and 34 so we sketch it here for completeness, and it is the proof that $\widetilde{g}(0)=\frac{1}{\rho} \frac{1}{(4 \pi A)^{3 / 2}}$. In the replica formalism $\widetilde{g}\left(\mathbf{x}, \mathbf{x}^{\prime}\right)=\rho^{-2} \sum_{i j}^{1, N}\langle\delta(\mathbf{x}$ $\left.\left.-\mathbf{x}_{i}^{a}\right) \delta\left(\mathbf{x}^{\prime}-\mathbf{x}_{j}^{b}\right)\right\rangle$, where $a \neq b$ are two different replicas. In the language of Refs. 33 and 34, replicas are arranged in molecules labeled by the index $i=1, \ldots, N$. Then if $i \neq j$ particles in different replicas are constrained to be at distance $\left|\mathbf{x}-\mathbf{x}^{\prime}\right| \gtrsim \sigma$ and do not contribute to $\widetilde{g}(\mathbf{x}, \mathbf{x})$. The only term that contributes is the one for $i=j$, and from Eq. (4) in Ref. 34 we have $\tilde{g}\left(\mathbf{x}, \mathbf{x}^{\prime}\right)$ $=\rho^{-2} \sum_{i=1}^{N}\left\langle\delta\left(\mathbf{x}-\mathbf{x}_{i}^{1}\right) \delta\left(\mathbf{x}^{\prime}-\mathbf{x}_{i}^{2}\right)\right\rangle=\rho^{-2} \int d \mathbf{x}_{3} \cdots d \mathbf{x}_{m} \rho\left(\mathbf{x}_{1}, \ldots, \mathbf{x}_{m}\right)$.

Then the result follows from Eqs. (11) and (12) of Ref. 34. 